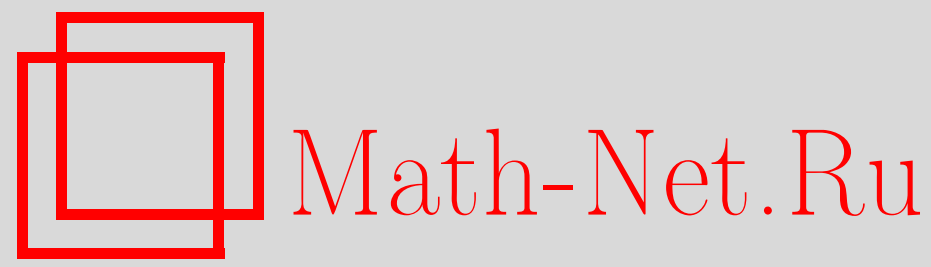

Л. Л. Максимова, Амальгамируемость, интерполяция и неявная определимость в многообразиях алгебр, Совр. пробл. матем., 2011, выпуск 15, 15-39

DOI: https://doi.org/10.4213/spm27

Использование Общероссийского математического портала Math-Net.Ru подразумевает, что вы прочитали и согласны с пользовательским соглашением http://www. mathnet.ru/rus/agreement

Параметры загрузки:

IP : 54.237 .59 .107

26 апреля 2023 г., 14:51:41 


\title{
Амальгамируемость, интерполяция и неявная определимость в многообразиях алгебр
}

\author{
Л. Л. Максимова \\ Институт математики им. С. Л. Соболева СО РАН, г. Новосибирск
}

\section{1. Введение}

Проблемы зависимости между структурными свойствами классов алгебр и свойствами формальных языков, описывающих эти классы, алгоритмические проблемы различных теорий играют большую роль в научном творчестве выдающегося математика Мальцева, основателя теории алгебраических систем [1], [2].

Алгебраическая семантика логических систем послужила основой для применения алгебраических методов в исследованиях по логике. К числу фундаментальных теорем логики относятся интерполяционная теорема Крейга [3] и теорема Бета о неявной определимости [4].

Мы рассматриваем проблемы амальгамируемости, интерполяции и справедливости свойства Бета и их вариантов в многообразиях алгебр. Нас интересует вопрос: когда исследование этих проблем можно свести к изучению более узких классов, а именно классов финитно неразложимых алгебр? Мы укажем достаточные условия для такого сведения.

В тех случаях, когда такое сведение возможно (например, в табличных конгруэнц-дистрибутивных многообразиях), получаем эффективный метод проверки исследуемых свойств. Метод применим для исследования свойств, близких к амальгамируемости, и интерполяционных свойств в многообразиях алгебр, ассоциированных с модальными и суперинтуиционистскими логиками, а также с подструктурными логиками и многими другими. Во многих случаях удается доказать разрешимость свойств на классе табличных логик и многообразий.

Взаимосвязи категорных свойств многообразий и синтаксических свойств их эквациональных теорий были обнаружены в 60-е годы XX века. Были установлены связи интерполяционного свойства с амальгамируемостью, позднее замечена связь свойства Бета с сюръективностью эпиморфизмов. Соотношения различных версий интерполяционного свойства и свойства Бета с амальгамируемостью и сюръективностью эпиморфизмов в алгебраической логике рассматривались в [5]-[14] и других работах.

Более подробно были изучены модальные и суперинтуиционистские логики (см. [15], [16]). Результаты о связи синтаксических и категорных свойств в подструктурных логиках представлены в [17].

При исследовании модальных логик обнаружилась возможность сведения интерполяционного свойства, свойства Бета и амальгамируемости в многообразиях модальных алгебр к подходящим свойствам классов финитно неразложимых алгебр. Это сведение имело большое значение для доказательства разрешимости указанных свойств в многообразиях гейтинговых алгебр и алгебр замыкания и в табличных многообразиях модальных алгебр. В этой статье будет получено обобщение указанных результатов о сведении на другие многообразия.

Работа выполнена при финансовой поддержке Российского фонда фундаментальных исследований (грант № 09-01-00090а), программы "Ведущие научные школы" (грант № НШ-3606.2010.1) и программы АВЦП Минобразования России "Развитие научного потенциала высшей школы" (грант № 2.1.1.10726).

(C) Максимова Л. Л., 2011 
Будут найдены достаточные условия для подобного сведения. Это позволит доказать разрешимость исследуемых свойств во многих табличных многообразиях, т.е. многообразиях, порожденных конечным множеством конечных алгебр.

В п. 2.1 приведены определения амальгамируемости и родственных свойств. В. 2.2 дан краткий обзор результатов об интерполяции, свойствах определимости по Бету и амальгамируемости в многообразиях алгебр, связанных с модальными, суперинтуиционистскими и позитивными логиками, приведены результаты о разрешимости указанных свойств. В параграфе 3 дан обзор результатов о взаимосвязях категорных и синтаксических свойств многообразий алгебр. Параграф 4 посвящен проблеме сведения вариантов амальгамируемости, интерполяции и свойства Бета к финитно неразложимым алгебрам. В параграфе 5 найдены достаточные условия разрешимости рассматриваемых свойств в табличных многообразиях алгебр.

\section{2. Основные сведения}

2.1. Определения. Пусть $V$ - класс алгебр. Напомним определения амальгамируемости AP, сверхамальгамируемости SupAP, сильной амальгамируемости StrAP, ограниченной амальгамируемости RAP, слабой амальгамируемости WAP, сюръективности эпиморфизмов $\mathrm{ES}^{*}$ и сильной сюръективности эпиморфизмов SES.

Класс $V$ амальгамируем, если он удовлетворяет условию

$\mathrm{AP}:$ Для любых $\mathbf{A}, \mathbf{B}, \mathbf{C} \in V$ таких, что $\mathbf{A}$ является общей подалгеброй алгебр $\mathbf{B}$ и $\mathbf{C}$, существуют алгебра $\mathbf{D}$ из $V$ и мономорфизмы $\delta: \mathbf{B} \rightarrow \mathbf{D}$ и $\varepsilon: \mathbf{C} \rightarrow \mathbf{D}$ такие, что $\delta(x)=\varepsilon(x)$ для всех $x \in \mathbf{A}$.

Класс $V$ сильно амальгамируем, если он удовлетворяет условию

StrAP : AP с дополнительным условием

$$
\delta(\mathbf{B}) \cap \varepsilon(\mathbf{C})=\delta(\mathbf{A}) .
$$

Класс $V$ обладает свойством ограниченной амальгамируемости, если выполнено условие $\mathrm{RAP}$ : Для любых $\mathbf{A}, \mathbf{B}, \mathbf{C} \in V$ таких, что $\mathbf{A}$ является общей подалгеброй алгебр $\mathbf{B}$ и $\mathbf{C}$, существуют алгебра $\mathbf{D}$ из $V$ и гомоморфизмы $\delta: \mathbf{B} \rightarrow \mathbf{D}$ и $\varepsilon: \mathbf{C} \rightarrow \mathbf{D}$ такие, что $\delta(x)=\varepsilon(x)$ для всех $x \in \mathbf{A}$ и ограничение $\delta^{\prime}$ отображения $\delta$ на $\mathbf{A}$ является мономорфизмом.

Класс $V$, в котором все алгебры частично упорядочены, называем сверхамальгамируемым, если он удовлетворяет условию

SupAP : AP с дополнительными условиями: для всех $x \in \mathbf{B}, y \in \mathbf{C}$

$$
\begin{aligned}
& \delta(x) \leqslant \varepsilon(y) \Longleftrightarrow(\exists z \in \mathbf{A})(x \leqslant z \text { и } z \leqslant y), \\
& \delta(x) \geqslant \varepsilon(y) \Longleftrightarrow(\exists z \in \mathbf{A})(x \geqslant z \text { и } z \geqslant y) .
\end{aligned}
$$

Рассмотрим также следующий слабый вариант свойства SupAP:

WSupAP : Если $\mathbf{A}$ - общая подалгебра алгебр $\mathbf{B}$ и $\mathbf{C}$ из $V$, то существуют $\mathbf{D}$ из $V$ и гомоморфизмы $\delta: \mathbf{B} \rightarrow \mathbf{D}, \varepsilon: \mathbf{C} \rightarrow \mathbf{D}$ такие, что $\delta(x)=\varepsilon(x)$ для всех $x \in \mathbf{A}$ и для всех $x \in \mathbf{B}, y \in \mathbf{C}$

$$
\begin{aligned}
& \delta(x) \leqslant \varepsilon(y) \Longleftrightarrow(\exists z \in \mathbf{A})(x \leqslant z \text { и } z \leqslant y), \\
& \delta(x) \geqslant \varepsilon(y) \Longleftrightarrow(\exists z \in \mathbf{A})(x \geqslant z \text { и } z \geqslant y) .
\end{aligned}
$$


Зафиксируем сигнатуру, состоящую из функциональных символов и констант и включающую две константы $\perp$ и Т. Алгебру $\mathbf{A}$ назовем ограниченной, если она удовлетворяет условию: $\mathbf{A}=\perp=\top$ тогда и только тогда, когда $\mathbf{A}$ одноэлементна. Рассмотрим многообразия, состоящие из ограниченных алгебр. В качестве примера можно взять ограниченные решетки, гейтинговы алгебры, модальные алгебры, булевы алгебры с операторами. Ограниченную алгебру называем невырожденной, если она содержит не менее двух элементов. Для многообразий ограниченных алгебр определим понятие слабой амальгамируемости.

WAP : для любых $\mathbf{A}, \mathbf{B}, \mathbf{C} \in V$ таких, что $\mathbf{A}$ есть общая подалгебра $\mathbf{B}$ и $\mathbf{C}$, существуют алгебра $\mathbf{D}$ из $V$ и гомоморфизмы $\delta: \mathbf{B} \rightarrow \mathbf{D}$ и $\varepsilon: \mathbf{C} \rightarrow \mathbf{D}$ такие, что $\delta(x)=\varepsilon(x)$ для всех $x \in \mathbf{A}$, где $\mathbf{D}$ невырожденная, если $\mathbf{A}$ невырожденная.

Определим также свойства сюргективности эпиморфизмов (ES*) и сильной сюргективности эпиморфизмов (SES).

$\mathrm{ES}^{*}$ : для любых $\mathbf{A}, \mathbf{B}$ из $V$, для любого мономорфизма $\alpha: \mathbf{A} \rightarrow \mathbf{B}$ и любого $x \in \mathbf{B}-\alpha(\mathbf{A})$ такого, что $\{x\} \cup \alpha(\mathbf{A})$ порождает $\mathbf{B}$, существуют $\mathbf{C} \in V$ и гомоморфизмы $\beta: \mathbf{B} \rightarrow \mathbf{C}$ и $\gamma: \mathbf{B} \rightarrow \mathbf{C}$ такие, что $\beta \alpha=\gamma \alpha$ и $\beta(x) \neq \gamma(x)$.

$\mathrm{SES}:$ для любых $\mathbf{A}, \mathbf{B}$ из $V$, для любого мономорфизма $\alpha: \mathbf{A} \rightarrow \mathbf{B}$ и любого $x \in \mathbf{B}-\alpha(\mathbf{A})$ существуют $\mathbf{C} \in V$ и гомоморфизмы $\beta: \mathbf{B} \rightarrow \mathbf{C}$ и $\gamma: \mathbf{B} \rightarrow \mathbf{C}$ такие, что $\beta \alpha=\gamma \alpha$ и $\beta(x) \neq \gamma(x)$.

В любых многообразиях алгебр имеем

$$
\mathrm{StrAP} \Longrightarrow \mathrm{AP} \Longrightarrow \mathrm{RAP} \Longrightarrow \mathrm{WAP}, \quad \mathrm{Str} A \mathrm{P} \Longrightarrow \mathrm{SES} \Longrightarrow \mathrm{ES}^{*} .
$$

В общем случае обратные импликации неверны. Кроме того,

$$
\mathrm{ES}^{*} \not \mathrm{WAP}, \quad \mathrm{AP} \not \mathrm{ES}^{*}, \quad \mathrm{StrAP} \Longleftrightarrow \mathrm{AP} \& \mathrm{ES}^{*}
$$

(см. теорему 3.14).

В многообразиях частично упорядоченных алгебр

$$
\text { SupAP } \Rightarrow \text { StrAP, } \quad \text { SupAP } \Rightarrow \text { WSupAP } \Rightarrow \text { SES. }
$$

Важную роль в наших рассмотрениях имеет свойство продолжаемости конгруэнций. Известно, что все многообразия модальных и гейтинговых алгебр обладают свойством продолжаемости конгруэниий.

$\mathrm{CEP}$ : для любых алгебр $\mathbf{A}, \mathbf{B} \in V$, где $\mathbf{A}$ является подалгеброй алгебры $\mathbf{B}$, любая конгруэнция $\Phi$ на $\mathbf{A}$ может быть продолжена до конгруэнции $\Psi$ на $\mathbf{B}$ такой, что ограничение отношения $\Psi$ на алгебру $\mathbf{A}$ совпадает с $\Phi$.

Обзоры по категорным свойствам классов алгебр, их соотношениям и взаимосвязям с синтаксическими свойствами теорий можно найти в [7], [11], [18]-[20]. Мы ограничимся несколькими примерами.

Многообразие $\mathscr{L}_{01}$ всех ограниченных решеток сверхамальгамируемо, а значит, имеет также StrAP, AP и SES [21]. С другой стороны, $\mathscr{L}_{01}$ не имеет CЕР.

Многообразие $\mathscr{D} \mathscr{L}$ дистрибутивных решеток имеет свойство продолжаемости конгруэнций СЕР [22]. Это верно и для дистрибутивных решеток с псевдодополнениями [23]. С другой стороны, отсутствие дистрибутивности часто влечет отсутствие свойства СЕР. Можно доказать, что единственное нетривиальное многобразие решеток со свойством СЕР - это многообразие дистрибутивных решеток. Многообразие решеток с псевдодополнениями не имеет СЕР [21].

Напомним, что решетка с псевдодополнениями - это ограниченная решетка с дополнительной операцией $\neg$, удовлетворяющая условию

$$
x \wedge y=\perp \Longleftrightarrow x \leqslant \neg y .
$$


Многообразие $\mathscr{D} \mathscr{P}$ всех дистрибутивных решеток с псевдодополнениями имеет свойство амальгамируемости AP [23]. Только три его собственных нетривиальных подмногообразия имеют АР [23], в частности, многообразие алгебр Стоуна (удовлетворяющих дополнительному тождеству $\neg x \vee \neg \neg x=1$ ) и многообразие булевых алгебр. Единственное нетривиальное подмногообразие многообразия $\mathscr{D} \mathscr{P}$ имеет SupAP и $\operatorname{StrAP}$ - это многообразие $\mathscr{B}$ булевых алгебр. Остальные подмногообразия не имеют ни $\mathrm{ES}^{*}$, ни SupAP, ни StrAP [21]. Все подмногообразия многообразия $\mathscr{D} \mathscr{P}$ имеют WAP [14].

2.2. Многообразия, ассоциированные с логиками. Алгебраическая семантика логических систем послужила основой для применения алгебраических методов в исследованиях по логике. K числу фундаментальных теорем логики относятся интерполяционная теорема Крейга [3] и теорема Бета о неявной определимости [4]. Имеется большое число работ по проблеме интерполяции и связанной с ней проблеме амальгамируемости в классах алгебр, ассоциированных с неклассическими логиками [6], [13], [15], [17].

Приведем краткий обзор результатов по проблеме амальгамируемости и свойствам Бета в многообразиях модальных и гейтинговых алгебр, а также близких к ним алгебр. Интерес к этой проблеме был вызван равносильностью интерполяционных свойств модальной или суперинтуиционистской логики и амальгамируемости соответствующего многообразия алгебр, обнаруженной в [15], [24], [25]. В частности, интуиционистская пропозициональная логика Int характеризуется многообразием гейтинговых алгебр, а любая суперинтуиционистская логика - подходящим подмногообразием этого многообразия. Суперинтуиционистская логика $L$ обладает интерполяционным свойством Крейга тогда и только тогда, когда соответствующее многообразие гейтинговых алгебр $V(L)$ амальгамируемо; кроме того, в случае гейтинговых алгебр амальгамируемость равносильна сверхамальгамируемости. Интерполяиионное свойство Крейга определяется следующим образом.

CIP : если в $L$ верна импликация $A \rightarrow B$, то существует интерполянт, т.е. такая формула $C$, что в $L$ верны $A \rightarrow C$ и $C \rightarrow B$ и все переменные формулы $C$ входят одновременно в $A$ и в $B$.

В модальных логиках свойство СІР эквивалентно сверхамальгамируемости соответствующего многообразия модальных алгебр, а амальгамируемость равносильна дедуктивному интерполяционному свойству.

IPD : если $A \vdash_{L} B$, то существует такая формула $C$, что $A \vdash_{L} C$ и $C \vdash_{L} B$ и все переменные формулы $C$ входят одновременно в $A$ и в $B$.

Свойства сюръективности эпиморфизмов SES и $\mathrm{ES}^{*}$ в модальных и суперинтуиционистских логиках равносильны [26], [15] соответственно свойствам Бета РВ2 и В2:

$\mathrm{PB} 2$ : пусть $\mathbf{x}, \mathbf{q}, \mathbf{q}^{\prime}$ - попарно не пересекающиеся списки переменных, не содержащие $y$ и $z, A(\mathbf{x}, \mathbf{q}, y)$ - формула. Если $A(\mathbf{x}, \mathbf{q}, y), A\left(\mathbf{x}, \mathbf{q}^{\prime}, z\right) \vdash_{L}(y \leftrightarrow z)$, то $A(\mathbf{x}, \mathbf{q}, y) \vdash_{L}(y \leftrightarrow B(\mathbf{x}))$ для некоторой формулы $B(\mathbf{x})$.

$\mathrm{B} 2$ : удаляя $\mathbf{q}$ и $\mathbf{q}^{\prime}$ из РВ2, получаем свойство В2.

Напомним некоторые определения. Импликативная решетка (или решетка с относительными псевдодополнениями $)$ - это алгебра $\mathbf{A}=(A, \wedge, \vee, \rightarrow, \top)$ с решеточными операциями $\wedge, \vee$, наибольшим элементом $T$ и дополнительной операцией относительного псевдодополнения, т.е.

$$
z \leqslant x \rightarrow y \Longleftrightarrow z \wedge x \leqslant y .
$$

Алгебра $\mathbf{A}=(A, \wedge, \vee, \rightarrow, \top, \perp)$, где $\mathbf{A}=(A, \wedge, \vee, \rightarrow, \top)$ есть импликативная решетка, a $\perp-$ произвольный элемент множества $A$, называется J-алгеброй. Гейтингова алгебра это Ј-алгебра, в которой $\perp$ является наименьшим элементом.

Модальной алгеброй называется алгебра $\mathbf{A}=(A, \wedge, \neg, \top, \square)$, где $(A, \wedge, \neg, \top)$ есть булева алгебра, а одноместная операция $\square$ удовлетворяет условиям

$$
\square \top=\top, \quad \square(x \wedge y)=\square x \wedge \square y .
$$


Модальная алгебра называется транзитивной, если на ней выполнено неравенство

$$
\square x \leqslant \square \square x \text {. }
$$

Транзитивная модальная алгебра называется топобулевой алгеброй, если удовлетворяет $\square x \leqslant x$, и диагонализируемой алгеброй, если

$$
\square(\square x \rightarrow x) \leqslant \square x, \quad \text { где } \quad y \rightarrow z=\neg(y \wedge \neg z) .
$$

Топобулева алгебра называется эпистемической, если выполнено

$$
x \leqslant \square \diamond x, \quad \text { где } \quad \diamond x=\neg \square \neg x,
$$

и гжегорчиковой алгеброй, если

$$
\square(\square(x \rightarrow \square x) \rightarrow x) \leqslant x
$$

Известно, что все указанные многообразия имеют свойство СЕР. Многообразия всех импликативных решеток, Ј-алгебр и гейтинговых алгебр, булевых алгебр, а также всех модальных алгебр, транзитивных модальных алгебр, топобулевых, диагонализируемых, эпистемических и гжегорчиковых алгебр сверхамальгамируемы. Кроме того, имеется лишь конечное число амальгамируемых многообразий топобулевых алгебр [27], [15] и конечное число амальгамируемых многообразий гейтинговых алгебр [24], хотя семейства многообразий топобулевых и гейтинговых алгебр имеют мощность континуума. Напротив, существует континуум амальгамируемых многообразий диагонализируемых алгебр [15]. При этом семейство неамальгамируемых многообразий диагонализируемых алгебр также имеет мощность континуума. Слабая амальгамируемость существенно отличается от ограниченной амальгамируемости и, тем более, от амальгамируемости. В частности, все многообразия диагонализируемых алгебр, гжегорчиковых и гейтинговых алгебр слабо амальгамируемы [14]. С другой стороны для класса эпистемических алгебр слабая амальгамируемость эквивалентна сильной амальгамируемости [28], поэтому все свойства, кроме $\mathrm{ES}^{*}$, равносильны.

В многообразиях модальных алгебр имеет место [12], [14], [15], [21]

$$
\begin{aligned}
\text { SupAP } \Longleftrightarrow \text { WSupAP } \Longrightarrow \mathrm{StrAP} \Longrightarrow \mathrm{AP} \Longrightarrow \mathrm{RAP} \Longrightarrow \mathrm{WAP}, \\
\text { StrAP } \Longleftrightarrow \mathrm{AP} \& \mathrm{ES}^{*} \Longrightarrow \mathrm{SES} \Longrightarrow \mathrm{ES}^{*} .
\end{aligned}
$$

В общем случае обратные импликации неверны. Кроме того,

$$
\mathrm{ES}^{*} \not \text { WAP. } \quad \mathrm{AP} \not \mathrm{ES}^{*}
$$

Все многообразия модальных транзитивных алгебр имеют $\mathrm{ES}^{*}$ [29], [15]. В многообразиях модальных транзитивных алгебр справедливы соотношения

$$
\begin{aligned}
& \text { SupAP } \Longleftrightarrow \text { WSupAP } \Longrightarrow \text { StrAP } \Longleftrightarrow \mathrm{AP} \Longrightarrow \mathrm{SES} \Longrightarrow \mathrm{RAP} \Longrightarrow \text { WAP }, \\
& \text { StrAP } \Longrightarrow \text { SupAP, SES } \Longrightarrow \mathrm{AP}, \quad \text { WAP } \Longrightarrow \mathrm{RAP} .
\end{aligned}
$$

В многообразиях эпистемических алгебр [28]:

$$
\text { SupAP } \Longleftrightarrow \mathrm{StrAP} \Longleftrightarrow \mathrm{AP} \Longleftrightarrow \mathrm{SES} \Longleftrightarrow \mathrm{RAP} \Longleftrightarrow \mathrm{WAP} .
$$

Для многообразий гейтинговых и гжегорчиковых алгебр и импликативных решеток имеем [30], [31]: 
все указанные многообразия имеют $\mathrm{ES}^{*}$ [15]; как было сказано выше, все многообразия гейтинговых и гжегорчиковых алгебр имеют WAP [14].

Полностью описаны многообразия гейтинговых и гжегорчиковых алгебр и импликативных решеток со свойствами амальгамируемости и сильной сюръективности эпиморфизмов [32]-[34]. В [15] указан список всех амальгамируемых многообразий топобулевых алгебр; установлено, что число амальгамируемых многообразий топобулевых алгебр не более 49 и не менее 43; все они конечно базируемы и порождаются своими конечными алгебрами. Найден эффективный критерий слабой амальгамируемости многообразий транзитивных модальных алгебр [35].

Найденные описания позволили доказать разрешимость алгоритмических проблем, связанных с интерполяцией и свойствами Бета в важных классах неклассических логик, с одной стороны, и с амальгамируемостью и сюръективностью эпиморфизмов в многообразиях соответствующих алгебр - с другой. Более точно, следующие свойства являются разрешимыми:

- интерполяционное свойство Крейга и дедуктивное интерполяционное свойство в суперинтуиционистских и позитивных исчислениях, а также в модальных исчислениях, содержащих модальную логику S4 [24], [27], [15];

- амальгамируемость, сильная амальгамируемость и сверхамальгамируемость в многообразиях топобулевых и гейтинговых алгебр и импликативных решеток [24], [27], [15];

- проективное свойство Бета над интуиционистской логикой и над логикой Гжегорчика, а также в позитивных логиках [36], [34], [33];

- сильная сюръективность эпиморфизмов и ограниченная амальгамируемость в многообразиях гейтинговых и гжегорчиковых алгебр и импликативных решеток [36], [34], [30], [33], [31];

- слабое интерполяционное свойство над модальной логикой K4 [35];

- слабая амальгамируемость в многообразиях транзитивных модальных алгебр [35].

Под разрешимостью свойства Р над логикой $L$ мы понимаем существование алгоритма, который по любой конечной системе схем аксиом, добавляемых к стандартным аксиомам и правилам логики $L$, определяет, обладает ли полученное в итоге исчисление свойством Р. Свойство Р считается разрешимым, например, в многообразиях гейтинговых алгебр, если существует алгоритм, который по любой конечной системе тождеств определяет, обладает ли свойством Р подмногообразие гейтинговых алгебр, удовлетворяющих этим тождествам.

Все указанные выше свойства являются разрешимыми также в табличных модальных, суперинтуиционистских и позитивных логиках и соответствующих многообразиях [37] (см. далее теорему 5.1). Логика (или многобразие) называется табличной, если порождается конечным числом конечных алгебр. Под разрешимостью свойства Р в табличных логиках понимается существование алгоритма, который по любой заданной конечной системе конечных алгебр определяет, обладает ли свойством Р логика, порожденная этой системой.

\section{3. Интерполяция, амальгамируемость и свойства Бета}

В этом параграфе будет дан обзор результатов о взаимосвязях категорных и синтаксических свойств многообразий алгебр. Свойство амальгамируемости классов алгебр связано с интерполяционными свойствами их эквационаьных теорий. Различные варианты свойства Бета равносильны подходящим вариантам свойства сюръективности эпиморфизмов. Соотношения различных версий интерполяционного свойства и свойства Бета с амальгамируемостью и сюръективностью эпиморфизмов в алгебраической логике рассматривались в [5], [7]-[12], [14], [15], [18] и других работах.

Приведем ряд определений. Зафиксируем произвольную сигнатуру, состоящую из функциональных символов и/или констант. Для данного множества переменных х через $t(\mathbf{x})$ обозначим любой терм, все переменные которого входят в $\mathbf{x}$, а через $F(\mathbf{x})$ - множество всех таких 
термов. Для данной алгебры А означиванием в А называется любой гомоморфизм алгебры термов в алгебру А. Если $t=t^{\prime}$ - равенство двух термов и $v$ - означивание, говорим, что $t=t^{\prime}$ выполнено в $\mathbf{A}$ при означивании $v$ и пишем $\mathbf{A} \models\left(t=t^{\prime}\right)[v]$, если $v(t)=v\left(t^{\prime}\right)$.

Если $\Gamma$ - множество равенств и $\gamma$ - равенство, то для любого многообразия $V$ определим:

$$
\Gamma \models_{V} \gamma \Longleftrightarrow(\forall \mathbf{A} \in V)(\forall v)(\mathbf{A}=\Gamma[v] \Longrightarrow \mathbf{A}=\gamma[v])
$$

Отметим, что отношение $\models_{V}$ можно заменить на синтаксическое отношение выводимости в эквациональной логике. Поэтому отношение $\models_{V}$ является компактным, т.е.

$$
\Gamma \models_{V} \gamma, \text { если и только если } \Gamma_{0} \models_{V} \gamma \text { для некоторого конечного } \Gamma_{0} \subseteq \Gamma \text {. }
$$

Для доказательств используется представление алгебр с помощью множества порождающих и определяющих соотношений [2].

Пусть фиксирована сигнатура $\Sigma$, состоящая из функциональных символов и/или констант. Если $\mathbf{x}$ - множество переменных, через $F(\mathbf{x})$ обозначаем множество всех термов данной сигнатуры от переменных из $\mathbf{x}$. Пусть заданы многообразие $V$ сигнатуры $\Sigma$ и алгебра $\mathbf{A} \in V$. Зафиксируем некоторое множество $X$ порождающих алгебры $\mathbf{A}$. Каждому элементу $a \in X$ ставим в соответствие переменную $p_{a}$, обозначим $\mathbf{x}=\left\{p_{a} \mid a \in X\right\}$. Определяем каноническое означивание $v_{0}\left(p_{a}\right)=a$. На множестве $F(\mathbf{x})$ определим отношение

$$
t={ }_{\mathbf{A}} t^{\prime} \Longleftrightarrow \mathbf{A} \models v_{0}(t)=v_{0}\left(t^{\prime}\right)
$$

ПРЕДЛОЖЕНИЕ 3.1. Отношение $=\mathbf{A}$ есть конгруэнция на $F(\mathbf{x})$ и существует изоморбизм $\varphi_{0}$ между $F(\mathbf{x}) /=\mathbf{A}$ и $\mathbf{A}$ такой, что $\varphi_{0}(t /=\mathbf{A})=v_{0}(t)$ для любого $t \in F(\mathbf{x})$.

Для данной алгебры $\mathbf{A}$ с множеством порождающих $X$ обозначаем

$$
D^{+}(\mathbf{A}, X)=\left\{\delta(\mathbf{x}) \mid \mathbf{A} \models \delta(\mathbf{x})\left[v_{0}\right]\right\} .
$$

Если $X$ совпадает с $\mathbf{A}$, то обозначаем

$$
D^{+}(\mathbf{A})=D^{+}(\mathbf{A}, X)
$$

Стандартным способом доказываются следующие утверждения.

ПрЕДЛОЖЕНИЕ 3.2. Если $\mathbf{B}=D^{+}(\mathbf{A}, X)[v]$ для некоторой алгебръ $\mathbf{B}$ и означивания $v$, то отображение $h(a)=v\left(p_{a}\right)$ можно продолжить до гомоморфизма из $\mathbf{A}$ в $\mathbf{B}$.

Если $\Theta$ - конгруэнция на $\mathbf{A}$, то запись $\left(a=a^{\prime}\right) \in \Theta$ означает, что $\left(a, a^{\prime}\right) \in \Theta$.

ПреДЛОЖЕНИЕ 3.3. Пусть $V$ - многообразие. Тогда $\Gamma \models_{V} \gamma$, если и толъко если для любой алгебры $\mathbf{A} \in V$, для любого означивания $v$ в $\mathbf{A}$ и для любой конгруэнции $\Theta$ на $\mathbf{A} и з$ $\Gamma[v] \subseteq \Theta$ следует $\gamma[v] \in \Theta$.

ПреДЛОЖЕНИЕ 3.4. Пусть $V$ - многообразие, $\mathbf{A} \in V, X-$ множество порождающих алгебры $\mathbf{A}, \Gamma \cup\{\delta\}$ - некоторое множество равенств от переменных из $\mathbf{x}=\left\{p_{a} \mid a \in X\right\}$, $\Phi$ - конгруэниия на $\mathbf{A}$, порожденная множеством $v_{0}(\Gamma)$. Тогда

$$
v_{0}(\delta) \in \Phi \Longleftrightarrow D^{+}(\mathbf{A}, X), \Gamma \models_{V} \delta \text {. }
$$

В частности, для любъх термов $t=t(\mathbf{x}), t^{\prime}=t^{\prime}(\mathbf{x})$ выполняется

$$
v_{0}(t)=\left.v_{0}\left(t^{\prime}\right) \Longleftrightarrow D^{+}(\mathbf{A}, X)\right|_{V} t=t^{\prime} .
$$

Вторая часть утверждения следует из первой при пустом Г. 
3.1. Амальгамируемость и интерполяция. Определим ряд интерполяционных свойств. Пусть $\mathbf{x}, \mathbf{y}, \mathbf{z}$ - попарно не пересекающиеся списки переменных, $\Gamma(\mathbf{x}, \mathbf{y}), \Delta(\mathbf{x}, \mathbf{z})-$ произвольные множества равенств, $\delta(\mathbf{x}, \mathbf{z})$ - равенство. Следующее свойство (интерполящионныи приниип для равенств) рассматривалось в [38]:

$\mathrm{EIP} \mathrm{:} \mathrm{если} \Gamma(\mathbf{x}, \mathbf{y}), \Delta(\mathbf{x}, \mathbf{z}) \models_{V} \delta(\mathbf{x}, \mathbf{z})$ и $F(\mathbf{x}) \neq \varnothing$, то существует $\Gamma^{\prime}(\mathbf{x})$ такое, что $\Gamma(\mathbf{x}, \mathbf{y}) \models_{V} \gamma$ для всех $\gamma \in \Gamma^{\prime}(\mathbf{x})$ и $\Gamma^{\prime}(\mathbf{x}), \Delta(\mathbf{x}, \mathbf{z}) \models_{V} \delta(\mathbf{x}, \mathbf{z})$.

В добавление мы определим свойство IPR ограниченной интерполячии:

$\mathrm{IPR}$ : если $\Gamma(\mathbf{x}, \mathbf{y}), \Delta(\mathbf{x}, \mathbf{z}) \models_{V} \delta(\mathbf{x})$, то существует $\Gamma^{\prime}(\mathbf{x})$ такое, что $\Gamma(\mathbf{x}, \mathbf{y}) \models_{V} \gamma$ для всех $\gamma \in \Gamma^{\prime}(\mathbf{x})$ и $\Gamma^{\prime}(\mathbf{x}), \Delta(\mathbf{x}, \mathbf{z}) \models_{V} \delta(\mathbf{x})$.

Ясно, что IPR - частный случай EIP.

Хотя IPR не симметрично, из него выводится симметричная форма IPR:

если $\Gamma(\mathbf{x}, \mathbf{y}), \Delta(\mathbf{x}, \mathbf{z}) \models_{V} \delta(\mathbf{x})$, то существуют $\Gamma^{\prime}(\mathbf{x})$ и $\Delta^{\prime}(\mathbf{x})$ такие, что $\Gamma(\mathbf{x}, \mathbf{y}) \models_{V} \gamma$ для всех $\gamma \in \Gamma^{\prime}(\mathbf{x}), \Delta(\mathbf{x}, \mathbf{z}) \models_{V} \delta$ для всех $\delta \in \Delta^{\prime}(\mathbf{x})$ и $\Gamma^{\prime}(\mathbf{x}),\left.\Delta^{\prime}(\mathbf{x})\right|_{V} \delta(\mathbf{x})$.

Для вывода достаточно применить IPR еще раз к выражению $\Gamma^{\prime}(\mathbf{x}), \Delta(\mathbf{x}, \mathbf{z}) \models_{V} \delta(\mathbf{x})$, поменяв местами $\Gamma^{\prime}(\mathbf{x}), \Delta(\mathbf{x}, \mathbf{z})$.

Рассмотрим также частный случай IPR при пустом $\mathbf{z}$, a именно,

WRIP : если $\Gamma(\mathbf{x}, \mathbf{y}), \Delta(\mathbf{x}) \models_{V} \delta(\mathbf{x})$, то существует $\Gamma^{\prime}(\mathbf{x})$ такое, что $\Gamma(\mathbf{x}, \mathbf{y}) \models_{V} \gamma$ для всех $\gamma \in \Gamma^{\prime}(\mathbf{x})$ и $\Gamma^{\prime}(\mathbf{x}), \Delta(\mathbf{x}) \models_{V} \delta(\mathbf{x})$.

Для классов частично упорядоченных алгебр определим интерполяиионный приниип для неравенств:

IIP : пусть $\Gamma(\mathbf{x}, \mathbf{y})$ и $\Delta(\mathbf{x}, \mathbf{z})$ удовлетворяют условию: $\Gamma^{\prime}(\mathbf{x})=\Delta^{\prime}(\mathbf{x})$, где

$$
\Gamma^{\prime}(\mathbf{x})=\left\{\alpha(\mathbf{x}) \mid \Gamma(\mathbf{x}, \mathbf{y}) \models_{V} \alpha(\mathbf{x})\right\}, \quad \Delta^{\prime}(\mathbf{x})=\left\{\alpha(\mathbf{x}) \mid \Delta(\mathbf{x}, \mathbf{z}) \models_{V} \alpha(\mathbf{x})\right\} ;
$$

если $\Gamma(\mathbf{x}, \mathbf{y}), \Delta(\mathbf{x}, \mathbf{z}) \models_{V} u(\mathbf{x}, \mathbf{y}) \leqslant v(\mathbf{x}, \mathbf{z})$, то существует терм $t(\mathbf{x})$ такой, что

$$
\Gamma(\mathbf{x}, \mathbf{y}) \models_{V} u(\mathbf{x}, \mathbf{y}) \leqslant t(\mathbf{x}), \quad \Delta(\mathbf{x}, \mathbf{z}) \models_{V} t(\mathbf{x}) \leqslant v(\mathbf{x}, \mathbf{z}) .
$$

Слабое интерполяционное свойство WIP определяем для классов ограниченных алгебр:

$$
\text { если } \Gamma(\mathbf{x}, \mathbf{y}),\left.\Delta(\mathbf{x}, \mathbf{z})\right|_{V} \perp=\top \text {, то существует } \Gamma^{\prime}(\mathbf{x}) \text { такое, что } \Gamma(\mathbf{x}, \mathbf{y}) \models_{V} \gamma
$$

для всех $\gamma \in \Gamma^{\prime}(\mathbf{x})$ и $\Gamma^{\prime}(\mathbf{x}), \Delta(\mathbf{x}, \mathbf{z}) \models_{V} \perp=\top$.

Под ограниченной алгеброй понимаем частично упорядоченную алгебру с наименьшим и наибольшим элементами $\perp$ и $\top$. Ясно, что WIP является частным случаем IPR.

ТЕОРема 3.5. Для любого многообразия $V$ :

- EIP равносильно конбюнкции $\mathrm{AP} \& \mathrm{CEP}[38] ;$

- IPR равносильно конгюнкиии RAP \& CEP [12];

- если $V$ - многообразие ограниченных алгебр, то WAP \& CEP $\Rightarrow$ WIP $\Rightarrow$ WAP [14];

- CEP равносильно WRIP [8].

Как мы видим, в случае многообразий со свойством продолжаемости конгруэнций СЕР свойство амальгамируемости равносильно свойству ЕIP. Однако в общем случае это не так [8]. Следующий эквивалент амальгамируемости в произвольных многообразиях найден в [8] (где это свойство обозначено через $\left.\mathrm{ROB}^{*}\right)$ :

$\mathrm{EIP}^{*}:$ пусть $\Gamma(\mathbf{x}, \mathbf{y})$ и $\Delta(\mathbf{x}, \mathbf{z})$ удовлетворяют условию: $\Gamma^{\prime}(\mathbf{x})=\Delta^{\prime}(\mathbf{x})$, где

$$
\Gamma^{\prime}(\mathbf{x})=\left\{\alpha(\mathbf{x}) \mid \Gamma(\mathbf{x}, \mathbf{y}) \models_{V} \alpha(\mathbf{x})\right\}, \quad \Delta^{\prime}(\mathbf{x})=\left\{\alpha(\mathbf{x}) \mid \Delta(\mathbf{x}, \mathbf{z}) \models_{V} \alpha(\mathbf{x})\right\} ;
$$

если $\Gamma(\mathbf{x}, \mathbf{y}), \Delta(\mathbf{x}, \mathbf{z}) \models_{V} \tau(\mathbf{x}, \mathbf{z})$, то $\Delta(\mathbf{x}, \mathbf{z}) \models_{V} \tau(\mathbf{x}, \mathbf{z})$. 
Введем также следующие свойства:

$\mathrm{IPR}^{*}:$ пусть $\Gamma(\mathbf{x}, \mathbf{y})$ и $\Delta(\mathbf{x}, \mathbf{z})$ удовлетворяют условию: $\Gamma^{\prime}(\mathbf{x})=\Delta^{\prime}(\mathbf{x})$, где

$$
\Gamma^{\prime}(\mathbf{x})=\left\{\alpha(\mathbf{x}) \mid \Gamma(\mathbf{x}, \mathbf{y}) \models_{V} \alpha(\mathbf{x})\right\}, \quad \Delta^{\prime}(\mathbf{x})=\left\{\alpha(\mathbf{x}) \mid \Delta(\mathbf{x}, \mathbf{z}) \models_{V} \alpha(\mathbf{x})\right\} ;
$$

если $\Gamma(\mathbf{x}, \mathbf{y}), \Delta(\mathbf{x}, \mathbf{z}) \models_{V} \tau(\mathbf{x})$, то $\Delta(\mathbf{x}, \mathbf{z}) \models_{V} \tau(\mathbf{x})$;

$\mathrm{WIP}^{*}$ : пусть $\Gamma(\mathbf{x}, \mathbf{y})$ и $\Delta(\mathbf{x}, \mathbf{z})$ удовлетворяют условию: $\Gamma^{\prime}(\mathbf{x})=\Delta^{\prime}(\mathbf{x})$, где

$$
\begin{aligned}
& \Gamma^{\prime}(\mathbf{x})=\left\{\alpha(\mathbf{x}) \mid \Gamma(\mathbf{x}, \mathbf{y}) \models_{V} \alpha(\mathbf{x})\right\}, \quad \Delta^{\prime}(\mathbf{x})=\left\{\alpha(\mathbf{x}) \mid \Delta(\mathbf{x}, \mathbf{z}) \models_{V} \alpha(\mathbf{x})\right\} ; \\
& \text { если } \Gamma(\mathbf{x}, \mathbf{y}), \Delta(\mathbf{x}, \mathbf{z}) \models_{V} \perp=\top, \text { то } \Delta(\mathbf{x}, \mathbf{z}) \models_{V} \perp=\top .
\end{aligned}
$$

ТЕОРема 3.6. Для любого многообразия $V$ :

(1) AP равносильно $\mathrm{EIP}^{*}$;

(2) RAP равносильно $\mathrm{IPR}^{*}$;

(3) если $V$ - многообразие ограниченных алгебр, то WAP равносилъно WIP*.

ДокАзАтельство. (1) Доказано в [8]. Приведем доказательство для (2). Аналогично доказывается (3).

$\mathrm{RAP} \Rightarrow \mathrm{IPR}^{*}$. Пусть $\Gamma(\mathbf{x}, \mathbf{y})$ и $\Delta(\mathbf{x}, \mathbf{z})$ удовлетворяют условию: $\Gamma^{\prime}(\mathbf{x})=\Delta^{\prime}(\mathbf{x})$, где

$$
\begin{gathered}
\Gamma^{\prime}(\mathbf{x})=\left\{\alpha(\mathbf{x})|\Gamma(\mathbf{x}, \mathbf{y})|_{V} \alpha(\mathbf{x})\right\}, \quad \Delta^{\prime}(\mathbf{x})=\left\{\alpha(\mathbf{x})|\Delta(\mathbf{x}, \mathbf{z})|_{V} \alpha(\mathbf{x})\right\} \\
\Gamma(\mathbf{x}, \mathbf{y}),\left.\Delta(\mathbf{x}, \mathbf{z})\right|_{V} \tau(\mathbf{x}) .
\end{gathered}
$$

Докажем, что $\Delta(\mathbf{x}, \mathbf{z}) \models_{V} \tau(\mathbf{x})$.

На множестве термов $F(\mathbf{x}, \mathbf{y})$ определяем конгруэнцию

$$
t(\mathbf{x}, \mathbf{y}) \approx_{1} t^{\prime}(\mathbf{x}, \mathbf{y}) \Longleftrightarrow \Gamma(\mathbf{x}, \mathbf{y}) \models_{V} t(\mathbf{x}, \mathbf{y})=t^{\prime}(\mathbf{x}, \mathbf{y}),
$$

а на множестве $F(\mathbf{x}, \mathbf{z})$ - конгруэнцию

$$
t(\mathbf{x}, \mathbf{z}) \approx_{2} t^{\prime}(\mathbf{x}, \mathbf{z}) \Longleftrightarrow \Delta(\mathbf{x}, \mathbf{z}) \models_{V} t(\mathbf{x}, \mathbf{z})=t^{\prime}(\mathbf{x}, \mathbf{z}) .
$$

Положим $\mathbf{B}^{\prime}=F(\mathbf{x}, \mathbf{y}) / \approx_{1}, \mathbf{C}=F(\mathbf{x}, \mathbf{z}) / \approx_{2}$. Очевидно, $\mathbf{B}^{\prime}, \mathbf{C} \in V$.

Пусть $\mathbf{A}$ - подалгебра алгебры $\mathbf{C}$, порожденная множеством $\mathbf{x} / \approx_{2}$. Из условия $\Gamma^{\prime}(\mathbf{x})=$ $\Delta^{\prime}(\mathbf{x})$ следует, что $t(\mathbf{x}) \approx_{1} t^{\prime}(\mathbf{x}) \Longleftrightarrow t(\mathbf{x}) \approx_{2} t^{\prime}(\mathbf{x})$ для любых термов $t(\mathbf{x}), t^{\prime}(\mathbf{x})$, а значит, отображение $\varphi\left(t(\mathbf{x}) / \approx_{2}\right)=\left(t(\mathbf{x}) / \approx_{1}\right)$ является мономорфизмом из $\mathbf{A}$ в $\mathbf{B}^{\prime}$. Отображение $\varphi^{-1}$ можно продолжить до изоморфизма $\psi$ из $\mathbf{B}^{\prime}$ на подходящую алгебру $\mathbf{B}$ такую, что $\mathbf{A}$ является подалгеброй алгебры В. В силу RAP существуют алгебра $\mathbf{D}$ из $V$ и гомоморфизмы $\delta: \mathbf{B} \rightarrow \mathbf{D}$ и $\varepsilon: \mathbf{C} \rightarrow \mathbf{D}$ такие, что $\delta(x)=\varepsilon(x)$ для всех $x \in \mathbf{A}$ и ограничение $\varepsilon^{\prime}$ отображения $\varepsilon$ на $\mathbf{A}$ является мономорфизмом.

В алгебре $\mathbf{D}$ определим означивание $v$ :

$$
v(y)=\delta \psi\left(y / \approx_{1}\right) \quad \text { для } y \in \mathbf{x} \cup \mathbf{y}, \quad v(z)=\varepsilon\left(z / \approx_{2}\right) \quad \text { для } z \in \mathbf{x} \cup \mathbf{z} .
$$

Определение корректно, так как выполняется $\delta \psi\left(x / \approx_{1}\right)=\varepsilon\left(x / \approx_{2}\right)$ при $x \in \mathbf{x}$. По построению алгебры $\mathbf{B}$ получаем $\mathbf{D} \models \Gamma(\mathbf{x}, \mathbf{y})[v]$, а по построению алгебры $\mathbf{C}$ имеем $\mathbf{D} \models \Delta(\mathbf{x}, \mathbf{z})[v]$. Следовательно, $\mathbf{D} \models \tau(\mathbf{x})[v]$. Отсюда, так как $\varepsilon^{\prime}-$ мономорфизм, получаем $\mathbf{C} \models \tau(\mathbf{x})\left[v_{0}\right]$, где $v_{0}(x)=x / \approx_{2}$. По определению алгебры $\mathbf{C}$ отсюда следует, что $\Delta(\mathbf{x}, \mathbf{z}) \models_{V} \tau(\mathbf{x})$.

$\mathrm{IPR}^{*} \Rightarrow \mathrm{RAP}$. Пусть $\mathbf{A}, \mathbf{B}, \mathbf{C} \in V, \mathbf{A}$ является общей подалгеброй алгебр $\mathbf{B}$ и $\mathbf{C}$. Обозначим

$$
\mathbf{x}=\left\{p_{a} \mid a \in \mathbf{A}\right\}, \quad \mathbf{y}=\left\{p_{a} \mid a \in \mathbf{B}-\mathbf{A}\right\}, \quad \mathbf{z}=\left\{p_{a} \mid a \in \mathbf{C}-\mathbf{A}\right\} .
$$

На множестве $F(\mathbf{x}, \mathbf{y}, \mathbf{z})$ положим

$$
t \Theta t^{\prime} \Longleftrightarrow D^{+}(\mathbf{B}), D^{+}(\mathbf{C}) \models_{V} t=t^{\prime} .
$$


Тогда $\Theta$ является конгруэнцией на $F(\mathbf{x}, \mathbf{y}, \mathbf{z})$. Обозначим

$$
\mathbf{D}=F(\mathbf{x}, \mathbf{y}, \mathbf{z}) / \Theta
$$

и положим

$$
g(b)=p_{b} / \Theta \quad \text { для } b \in \mathbf{B}, \quad h(c)=p_{c} / \Theta \quad \text { для } c \in \mathbf{B} .
$$

Тогда по предложению $3.2 \mathrm{~g}, h$ - гомоморфизмы из $\mathbf{B}$ и $\mathbf{C}$ соответственно в алгебру $\mathbf{D}$, причем $g(a)=h(a)$ для всех $a \in \mathbf{A}$.

Покажем, что ограничение гомоморфизма $g$ на алгебру $\mathbf{A}$ является мономорфизмом. Пусть $a, b \in \mathbf{A}$ и $g(a)=g(b)$. Тогда по определению алгебры $\mathbf{D}$ получаем

$$
D^{+}(\mathbf{B}), D^{+}(\mathbf{C}) \models_{V} p_{a}=p_{b} .
$$

Поскольку $\mathbf{A}$ является общей подалгеброй алгебр $\mathbf{B}$ и $\mathbf{C}$, для любого равенства $\tau(\mathbf{x})$ выполняется

$$
D^{+}(\mathbf{B}) \models_{V} \tau(\mathbf{x}) \Longleftrightarrow D^{+}(\mathbf{C}) \models_{V} \tau(\mathbf{x}) .
$$

По IPR* получаем

$$
D^{+}(\mathbf{C}) \models{ }_{V} p_{a}=p_{b} .
$$

По предложению 3.4 отсюда следует $a=b$, что и требовалось.

В [21] найден алгебраический эквивалент для свойства IIP в многообразиях алгебр, основанных на решетках. Доказательство верно и для полурешеточно упорядоченных алгебр, т.е. алгебр, в сигнатуре которых присутствует операция $\wedge$, которая интерпретируется как точная нижняя грань.

ТеОРема 3.7 [21]. Пусть $V$ - многообразие полурешеточно упорядоченных алгебр. Тогда

- WSUpAP равносильно IIP;

- SupAP равносильно конбюнкиии IIP \& EIP* .

Поскольку существуют амальгамируемые многообразия без свойства SupAP, из теоремы 3.7 следует, что в общем случае IIP не следует из АР.

Рассмотрим случай, когда в рассматриваемом многообразии наряду с операцией $\wedge$ взятия точной нижней грани, существуют термы $\mathbf{e}(x, y)$ и $\mathbf{t}$ без переменных, определяющие равенство, т.е. удовлетворяющие условию

$$
\mathrm{EQ}: x=y \Longleftrightarrow \mathbf{t} \leqslant \mathbf{e}(x, y) \text {. }
$$

Примерами таких многообразий могут служить многообразия булевых алгебр с операторами, гейтинговых алгебр и алгебр, связанных с нечеткими, подструктурными и релевантными логиками.

ПРЕДЛОЖЕНИЕ 3.8. В многообразиях, удовлетворяющих условию EQ, выполнено

$$
\text { WSupAP } \Longrightarrow \text { SupAP. }
$$

Доказательство. Учитывая теорему 3.7 , выведем EIP* из IIP. Пусть $\Gamma(\mathbf{x}, \mathbf{y})$ и $\Delta(\mathbf{x}, \mathbf{z})$ удовлетворяют условию: $\Gamma^{\prime}(\mathbf{x})=\Delta^{\prime}(\mathbf{x})$, где

$$
\Gamma^{\prime}(\mathbf{x})=\left\{\alpha(\mathbf{x}) \mid \Gamma(\mathbf{x}, \mathbf{y}) \models_{V} \alpha(\mathbf{x})\right\}, \quad \Delta^{\prime}(\mathbf{x})=\left\{\alpha(\mathbf{x}) \mid \Delta(\mathbf{x}, \mathbf{z}) \models_{V} \alpha(\mathbf{x})\right\} .
$$

Пусть

$$
\Gamma(\mathbf{x}, \mathbf{y}), \Delta(\mathbf{x}, \mathbf{z}) \models_{V} t_{1}=t_{2}, \quad \text { где } \quad t_{1}=t_{1}(\mathbf{x}, \mathbf{z}), \quad t_{2}=t_{2}(\mathbf{x}, \mathbf{z}) .
$$

Докажем, что $\Delta(\mathbf{x}, \mathbf{z}) \models_{V} t_{1}=t_{2}$.

По условию $\mathrm{EQ}$ получаем

$$
\Gamma(\mathbf{x}, \mathbf{y}), \Delta(\mathbf{x}, \mathbf{z}) \models_{V} \mathbf{t} \leqslant \mathbf{e}\left(t_{1}, t_{2}\right) .
$$


По IIP существует $t(\mathbf{x})$ такой, что

$$
\Gamma(\mathbf{x}, \mathbf{y}) \models_{V} \mathbf{t} \leqslant t(\mathbf{x}), \quad \Delta(\mathbf{x}, \mathbf{z}) \models_{V} t(\mathbf{x}) \leqslant \mathbf{e}\left(t_{1}, t_{2}\right) .
$$

Отсюда $\Gamma(\mathbf{x}, \mathbf{y}) \models_{V} \mathbf{t} \wedge t(\mathbf{x})=\mathbf{t}$, а значит, $\Delta(\mathbf{x}, \mathbf{y}) \models_{V} \mathbf{t} \leqslant t(\mathbf{x})$, поскольку $\Gamma^{\prime}(\mathbf{x})=\Delta^{\prime}(\mathbf{x})$. Поэтому $\Delta(\mathbf{x}, \mathbf{z}) \models_{V} \mathbf{t} \leqslant \mathbf{e}\left(t_{1}, t_{2}\right)$ и $\Delta(\mathbf{x}, \mathbf{z}) \models_{V} t_{1}=t_{2}$.

Из этого предложения и теоремы 3.7 сразу вытекает

СлЕДСТВИЕ 3.9. Для любого многобразия, удовлетворяющего условию ЕQ, свойства IIP, SupAP $u$ WSupAP равносильны.

3.2. Свойства Бета и сюръективность эпиморфизмов. Пусть $\mathbf{x}, \mathbf{q}, \mathbf{q}^{\prime}-$ попарно не пересекающиеся списки переменных, не содержащие $y$ и $z, \Gamma(\mathbf{x}, \mathbf{q}, y)$ - некоторое множество формул. Говорим, что многообразие $V$ имеет проективное свойство Бета РВР, если выполнено условие:

$\Gamma(\mathbf{x}, \mathbf{q}, y), \Gamma\left(\mathbf{x}, \mathbf{q}^{\prime}, z\right) \models_{V} y=z$, где $F(\mathbf{x}) \neq \varnothing$ влечет $\Gamma(\mathbf{x}, \mathbf{q}, y) \models_{V} y=t(\mathbf{x})$ для некоторого терма $t(\mathbf{x})$.

Многообразие $V$ имеет свойство Бета ВР, если удовлетворяет условию:

из $\Gamma(\mathbf{x}, y), \Gamma(\mathbf{x}, z) \models_{V} y=z$, где $F(\mathbf{x}) \neq \varnothing$, следует $\left.\Gamma(\mathbf{x}, y)\right|_{V} y=t(\mathbf{x})$ для

некоторого терма $t(\mathbf{x})$.

Рассмотрим также финитарное свойство Бета FBP:

пусть $\mathbf{y}=\left(y_{1}, \ldots, y_{n}\right), \mathbf{z}=\left(z_{1}, \ldots, z_{n}\right)$ и для всех $1 \leqslant i \leqslant n$ выполнено

$\Gamma(\mathbf{x}, \mathbf{y}),\left.\Gamma(\mathbf{x}, \mathbf{z})\right|_{V} y_{i}=z_{i}$, где $F(\mathbf{x}) \neq \varnothing$. Тогда существуют термы $t_{i}(\mathbf{x})$ такие, что $\left.\Gamma(\mathbf{x}, y)\right|_{V} y_{i}=t_{i}(\mathbf{x})$ для всех $1 \leqslant i \leqslant n$.

Ясно, что $\mathrm{PBP} \Rightarrow \mathrm{FBP} \Rightarrow \mathrm{BP}$.

Заметим, что можно вывести FBP из ВР [26], [11].

ПРЕДЛОЖЕНИЕ 3.10. Для любого многообразия свойства FВР и ВР равносильнъ.

ДоказАТЕльство. Индукция по $n$. Пусть для всех $1 \leqslant i \leqslant n$ выполнено

$$
\Gamma\left(\mathbf{x}, y_{1}, \ldots, y_{n}\right), \Gamma\left(\mathbf{x}, z_{1}, \ldots, z_{n}\right) \models_{V} y_{i}=z_{i},
$$

где $F(\mathbf{x}) \neq \varnothing$. При $n=1$ требуемый терм существует по ВР.

Пусть $n>1$. Тогда по ВР существует терм $t\left(\mathbf{x}, y_{1}, \ldots, y_{n-1}\right)$ такой, что

$$
\Gamma\left(\mathbf{x}, y_{1}, \ldots, y_{n}\right) \models_{V} y_{n}=t\left(\mathbf{x}, y_{1}, \ldots, y_{n-1}\right) .
$$

Подставим в исходное соотношение $t\left(\mathbf{x}, y_{1}, \ldots, y_{n-1}\right)$ вместо $y_{n}$ и $t\left(\mathbf{x}, z_{1}, \ldots, z_{n-1}\right)$ вместо $z_{n}$. Получаем для всех $1 \leqslant i \leqslant n-1$

$$
\Gamma\left(\mathbf{x}, y_{1}, \ldots, y_{n-1}, t\left(\mathbf{x}, y_{1}, \ldots, y_{n-1}\right)\right), \Gamma\left(\mathbf{x}, z_{1}, \ldots, z_{n-1}, t\left(\mathbf{x}, z_{1}, \ldots, z_{n-1}\right)\right) \models_{V} y_{i}=z_{i} .
$$

По индукционной гипотезе для всех $i \leqslant n-1$ существуют термы $t_{i}(\mathbf{x})$ такие, что

$$
\left.\Gamma\left(\mathbf{x}, y_{1}, \ldots, y_{n-1}, t\left(\mathbf{x}, y_{1}, \ldots, y_{n-1}\right)\right)\right|_{V} y_{i}=t_{i}(\mathbf{x}) \text {. }
$$

Учитывая, что

$$
\Gamma\left(\mathbf{x}, y_{1}, \ldots, y_{n}\right), y_{n}=t\left(\mathbf{x}, y_{1}, \ldots, y_{n-1}\right) \models_{V} \Gamma\left(\mathbf{x}, y_{1}, \ldots, y_{n-1}, t\left(\mathbf{x}, y_{1}, \ldots, y_{n-1}\right)\right),
$$

и что равенство $y_{n}=t\left(\mathbf{x}, y_{1}, \ldots, y_{n-1}\right)$ выводится из $\Gamma\left(\mathbf{x}, y_{1}, \ldots, y_{n}\right)$, получаем, что для всех $i \leqslant n-1$ выполняется

$$
\Gamma\left(\mathbf{x}, y_{1}, \ldots, y_{n}\right) \models_{V} y_{i}=t_{i}(\mathbf{x})
$$

Следовательно,

$$
\Gamma\left(\mathbf{x}, y_{1}, \ldots, y_{n}\right) \models_{V} y_{n}=t\left(\mathbf{x}, t_{1}(\mathbf{x}), \ldots, t_{n-1}(\mathbf{x})\right)
$$


Известно, что свойство Бета не следует из интерполяционного свойства ЕIP: существует контрпример в многобразиях модальных алгебр [15]. Однако легко вывести РВР из IIP.

ПРЕДЛОЖенИЕ 3.11. Для любого многообразия частично упорядоченных алгебр проективное свойство Бета РВP следует из IIP.

ДокАЗАтЕльСтво. Пусть $\Gamma(\mathbf{p}, \mathbf{q}, x), \Gamma\left(\mathbf{p}, \mathbf{q}^{\prime}, y\right) \models_{V} x=y$. Тогда

$$
\Gamma(\mathbf{p}, \mathbf{q}, x), \Gamma\left(\mathbf{p}, \mathbf{q}^{\prime}, y\right) \models_{V} x \leqslant y .
$$

Ясно, что для любого $\delta(\mathbf{p})$ выполнено условие

$$
\Gamma(\mathbf{p}, \mathbf{q}, x) \models_{V} \delta(\mathbf{p}) \Longleftrightarrow \Gamma\left(\mathbf{p}, \mathbf{q}^{\prime}, y\right) \models_{V} \delta(\mathbf{p}) .
$$

По IIP существует терм $t(\mathbf{p})$ такой, что $\Gamma(\mathbf{p}, \mathbf{q}, x) \models_{V} x \leqslant t(\mathbf{p})$ и $\Gamma\left(\mathbf{p}, \mathbf{q}^{\prime}, y\right) \models_{V} t(\mathbf{p}) \leqslant y$. Отсюда $\Gamma(\mathbf{p}, \mathbf{q}, x) \models_{V} x=t(\mathbf{p})$.

Таким образом, в многообразиях частично упорядоченных алгебр выполнено

$$
\mathrm{IIP} \Longrightarrow \mathrm{PBP} \Longrightarrow \mathrm{FBP} \Longleftrightarrow \mathrm{BP} .
$$

Кроме того,

$$
\mathrm{PBP} \nRightarrow \mathrm{IIP}, \quad \mathrm{BP} \not \mathrm{PBP}
$$

(см. [32]).

Укажем алгебраические эквиваленты для свойств Бета.

Теорема 3.12. Для любого многообразия алгебр:

(1) проективное свойство Бета PBP равносильно SES;

(2) свойство Бета ВР равносильно ЕS*.

ДокАзАтельство. (1) Доказано в [26] для многообразий модальных и гейтинговых алгебр, доказательство легко переносится на общий случай [11].

(2) Доказано в [25].

Другой эквивалент свойства Бета ВР был найден ранее Немети [39, теорема 5.6.10]:

$\mathrm{RES}:$ пусть $\mathbf{A}, \mathbf{B} \in V$ и $h$ - гомоморфизм из $\mathbf{A}$ в $\mathbf{B}$, где $\mathbf{B}$ порождается множеством $h(\mathbf{A}) \cup\{a\}$ для некоторого $a \in \mathbf{B}$; если для любых гомоморфизмов $k$, $l$ алгебры $\mathbf{B}$ в $\mathbf{C} \in V$ выполняется $k h=l h \Rightarrow k=l$, то $h(\mathbf{A})=\mathbf{B}$.

В дополнение укажем эквивалент свойства Бета в терминах максимальных подалгебр:

ESM : если $\mathbf{A}$ - максимальная подалгебра алгебры $\mathbf{B} \in V$, то существуют $\mathbf{C} \in V$ и гомоморфизмы $g, h$ из $\mathbf{B}$ в $\mathbf{C}$ такие, что $g(x)=h(x)$ для всех $x \in \mathbf{A}$ и $g \neq h$.

ПрЕДЛОЖЕНИЕ 3.13. Для любого многообразия свойство ES* равносильно свойству ESM.

ДокАЗАтЕльство. $\Rightarrow$. Пусть $V$ имеет $\mathrm{ES}^{*}$ и $\mathbf{A}$ - максимальная подалгебра алгебры $\mathbf{B}$. Существует $b \in \mathbf{B}-\mathbf{A}$. Тогда $\mathbf{A} \cup\{b\}$ порождает $\mathbf{B}$ и по $\mathrm{ES}^{*}$ существуют $\mathbf{C} \in V$ и гомоморфизмы $g, h$ из $\mathbf{B}$ в $\mathbf{C}$ такие, что $g(x)=h(x)$ для всех $x \in \mathbf{A}$ и $g(b) \neq h(b)$.

$\Leftarrow$. Пусть $\mathbf{A}$ - подалгебра алгебры $\mathbf{B}, b \in \mathbf{B}-\mathbf{A}, \mathbf{A} \cup\{b\}$ порождает $\mathbf{B}$. Рассмотрим

$$
\Sigma=\left\{\mathbf{A}^{\prime} \mid \mathbf{A}^{\prime} \text { - подалгебра алгебры } \mathbf{B}, \mathbf{A} \subseteq \mathbf{A}^{\prime}, b \in \mathbf{B}-\mathbf{A}^{\prime}\right\} .
$$

Тогда $\Sigma$ удовлетворяет условиям леммы Цорна, а значит, имеет максимальный элемент $\mathbf{A}_{1}$. Покажем, что $\mathbf{A}_{1}$ - максимальная подалгебра алгебры В. Допустим, что собственная подалгебра $\mathbf{A}^{\prime}$ алгебры $\mathbf{B}$ содержит $\mathbf{A}_{1}$. Тогда $b \in \mathbf{B}-\mathbf{A}^{\prime}$, а значит, $\mathbf{A}^{\prime} \in \Sigma$ и $\mathbf{A}^{\prime}=\mathbf{A}_{1}$.

По ESM существуют $\mathbf{C} \in V$ и гомоморфизмы $g, h$ из $\mathbf{B}$ в $\mathbf{C}$ такие, что $g(x)=h(x)$ для всех $x \in \mathbf{A}_{1}$ и $g \neq h$. Поскольку $\mathbf{A}_{1} \cup\{b\}$ порождает $\mathbf{B}$, получаем $g(b) \neq h(b)$. 
Можно показать, что свойство ESM равносильно каждому из следующих свойств ESM1 и ESM2.

ESM1 : если $\mathbf{A}$ - максимальная подалгебра алгебры $\mathbf{B} \in V, b \in \mathbf{A}-\mathbf{B}$, то существуют $\mathbf{C} \in V$ и гомоморфизмы $g, h$ из $\mathbf{B}$ в $\mathbf{C}$ такие, что $g(x)=h(x)$ для всех $x \in \mathbf{A}$ и $g(b) \neq h(b)$.

ESM2: если $\mathbf{A}$ - максимальная подалгебра алгебры $\mathbf{B} \in V$, то существуют $\mathbf{C} \in V$ и гомоморфизмы $g, h$ из $\mathbf{B}$ в $\mathbf{C}$ такие, что $g(x)=h(x)$ для всех $x \in \mathbf{A}$ и $g(b) \neq h(b)$ для всех $b \in \mathbf{B}-\mathbf{A}$.

В многообразиях гейтинговых алгебр свойства SES и PBP следуют из амальгамируемости. Однако это нарушается в многообразиях модальных алгебр, где в общем случае ВР не следует из АР. В многообразиях модальных алгебр свойство сильной амальгамируемости StrAP равносильно конъюнкции АР \& ВР [25]. Это же справедливо и для произвольных многобразий [19], [17].

ТЕОРЕма 3.14. Для любого многообразия свойство силъной амальгамируемости StrAP равносильно конгюнкции $\mathrm{AP} \& \mathrm{ES}^{*}$ и влечет SES.

Доказательство. 1) StrAP равносильно АP \& ES* [25], [19], [17].

2) Свойство SES следует из StrAP: достаточно в определении $\operatorname{StrAP}$ подожить $\mathbf{C}=\mathbf{B}$.

Свойство ограниченной амальгамируемости RAP следует из SES в многообразиях гейтинговых алгебр [32]. Следующая теорема применима также к произвольным многообразиям модальных алгебр, решеток с относительными псевдодополнениями, а также к многообразиям резидуальных решеток [40].

Напомним, что алгебра называется невырожденной, если она содержит не менее двух элементов. Невырожденная алгебра называется подпрямо неразложимой, если она не представима как подпрямое произведение ее собственных факторалгебр. Алгебра называется финитно неразложимой, если не представима как конечное подпрямое произведение ее собственных факторалгебр.

ТеОРема 3.15 [12]. Пусть многообразие $V$ конгруэни-дистрибутивно и, кроме того, подалгебры подпрямо неразложимых алгебр из $V$ финитно неразложимы. Тогда если $V$ имеет SES, mo $V$ uмeеm RAP.

Учитывая теоремы 3.5 и 3.12 , получаем

СЛЕДСТВИЕ 3.16. Пусть многообразие $V$ конгруэни-дистрибутивно и, кроме того, подалгебры подпрямо неразложимых алгебр финитно неразложимы. Тогда если $V$ имеет CEP u PBP, то оно обладает свойством IPR.

Эти результаты можно применить к известным классам алгебр. Суммируя полученные утверждения и учитывая результаты из [25], [26], для многообразий модальных алгебр мы получаем следующие соотношения:

1) IIP $\Leftrightarrow$ SupAP;

2) $\mathrm{IIP} \Rightarrow \mathrm{EIP} \& \mathrm{BP} \Leftrightarrow \mathrm{Str} A \mathrm{P}, \mathrm{EIP} \& \mathrm{BP} \nRightarrow \mathrm{IIP}$;

3) $\mathrm{EIP} \& \mathrm{BP} \Rightarrow \mathrm{PBP} \Leftrightarrow \mathrm{SES}$;

4) $\mathrm{PBP} \nRightarrow \mathrm{EIP} \Leftrightarrow \mathrm{AP}, \mathrm{EIP} \nRightarrow \mathrm{PBP}$;

5) $\mathrm{PBP} \Rightarrow \mathrm{IPR} \Leftrightarrow \mathrm{RAP}$;

6) $\mathrm{EIP} \Rightarrow \mathrm{IPR}, \mathrm{IPR} \& \mathrm{BP} \nRightarrow \mathrm{EIP}$;

7) $\mathrm{PBP} \Rightarrow \mathrm{BP}, \mathrm{IPR} \nRightarrow \mathrm{BP}, \mathrm{BP} \nRightarrow \mathrm{IPR}$.

Аналогичные соотношения имеют место для многообразий резидуальных решеток [40], так как они обладают свойством СЕР, а также удовлетворяют условиям теоремы 3.15. 


\section{4. Сведение к финитно неразложимым алгебрам}

При исследовании модальных логик обнаружилась возможность сведения интерполяционного свойства, свойства Бета и амальгамируемости в многообразиях модальных алгебр к подходящим свойствам классов финитно неразложимых алгебр. Это сведение имело большое значение для доказательства разрешимости указанных свойств в многообразиях гейтинговых алгебр и алгебр замыкания и в табличных многообразиях модальных алгебр. В этом параграфе будет получено обобщение указанных результатов о сведении на другие многообразия. Будут найдены достаточные условия для подобного сведения.

Для сравнения приведем теорему о финитизации для многобразий модальных алгебр и родственных многообразий.

ТеоремА 4.1. Для любого многообразия $V$ модалъных алгебр, Ј-алгебр или импликативных решеток:

(1) $V$ имеет SupAP тогда и только тогда, когда для любых конечно порожденных фбнитно неразложимых $\mathbf{A}, \mathbf{B}, \mathbf{C} \in V$, мономорфизмов $\beta: \mathbf{A} \rightarrow \mathbf{B}, \gamma: \mathbf{A} \rightarrow \mathbf{C}$ и элементов $b_{0} \in \mathbf{B}, c_{0} \in \mathbf{C}$ таких, что не существует $a \in \mathbf{A}$, удовлетворяющего условиям $b_{0} \leqslant \beta(a)$ u $\gamma(a) \leqslant c_{0}$, существуют подпрямо неразложимая алгебра $\mathbf{D} \in V u$ гомоморфизмы $g: \mathbf{B} \rightarrow \mathbf{D} u h: \mathbf{C} \rightarrow \mathbf{D}$ maкue, чmo $g \beta=h \gamma$ u $g\left(b_{0}\right) \nless{ }^{\prime}\left(c_{0}\right)$ [24], [25], [33];

(2) $V$ имеет AP тогда и только тогда, когда для любых конечно порожденных финитно неразложимых $\mathbf{A}, \mathbf{B}, \mathbf{C} \in V$, мономорфизмов $\beta: \mathbf{A} \rightarrow \mathbf{B}, \gamma: \mathbf{A} \rightarrow \mathbf{C}$ и элемента $c_{0} \in \mathbf{C}$, $c_{0} \neq \top$, существуют подпрямо неразложимая алгебра $\mathbf{D}$ из $V$ и гомоморфизмы $\delta: \mathbf{B} \rightarrow$ $\mathbf{D}, \varepsilon: \mathbf{C} \rightarrow \mathbf{D}$ maкие, что $\delta \beta(x)=\varepsilon \gamma(x)$ для всех $x \in \mathbf{A} u \delta\left(c_{0}\right) \neq \top$ [24], [25], [33];

(3) $V$ uмеет StrAP тогда и только тогда, когда $V$ имеет AP $u$ ES* [25];

(4) $V$ имеет RAP тогда и только тогда, когда для любых конечно порожденных подпрямо неразложимых $\mathbf{A}, \mathbf{B}, \mathbf{C} \in V$, мономорфизмов $\beta: \mathbf{A} \rightarrow \mathbf{B}, \gamma: \mathbf{A} \rightarrow \mathbf{C}$ и различных элементов $a_{1}, a_{2} \in \mathbf{A}$ существуют подпрямо неразложимая алгебра $\mathbf{D} \in V$ и гомоморбизмы $g: \mathbf{B} \rightarrow \mathbf{D} u h: \mathbf{C} \rightarrow \mathbf{D}$ maкuе, чmо $g \beta=h \gamma$ u $g \beta\left(a_{1}\right) \neq h \gamma\left(a_{2}\right)$ [41];

(5) многообразие $V$ модальных или гейтинговых алгебр имеет WAP тогда и только тогда, когда класс конечно порожденных финитно неразложимых алгебр из V имеет WAP [14];

(6) $V$ имеет $\mathrm{ES}^{*}$ тогда и только тогда, когда класс конечно порожденных финитно неразложимых алгебр из $V$ имеет $\mathrm{ES}^{*}$ [25];

(7) $V$ имеет SES тогда и только тогда, когда для любых финитно неразложимых конечно порожденных $\mathbf{A}, \mathbf{B}, \mathbf{C}$ из $V$, для любых мономорфизмов $\beta: \mathbf{A} \rightarrow \mathbf{B}, \gamma: \mathbf{A} \rightarrow \mathbf{C} u д_{л я}$ любых $x \in \mathbf{B}, y \in \mathbf{C}$, если $\neg(\exists z \in \mathbf{A})(x=\beta(z) \& \gamma(z)=y)$, то существуют подпрямо неразложимая алгебра $\mathbf{D}$ из $V(L)$ и гомоморбизмы $\delta: \mathbf{B} \times \mathbf{C} \rightarrow \mathbf{D}, \varepsilon: \mathbf{B} \times \mathbf{C} \rightarrow \mathbf{D}$ такие, что $\delta(x, y) \neq \varepsilon(x, y)$ и $\delta(\beta(z), \gamma(z))=\varepsilon(\beta(z), \gamma(z))$ для всех z из $\mathbf{A}[26]$.

Напомним, что алгебра называется невырожденной, если она содержит не менее двух элементов. Невырожденная алгебра подпрямо неразложима, если она не представима как подпрямое произведение ее собственных фактор-алгебр. Алгебра бинитно неразложима, если не представима как конечное подпрямое произведение ее собственных фактор-алгебр. Невырожденная алгебра А называется простой, если она имеет точно две конгруэнции: нулевую конгруэнцию $\operatorname{id}_{\mathbf{A}}=\{(a, a) \mid a \in \mathbf{A}\}$ и несобственную конгруэнцию $\mathbf{A}^{2}$.

Для данного класса алгебр $V$ через $\mathrm{SI}(V)$ обозначается класс подпрямо неразложимых, через $\mathrm{FI}(V)$ - класс финитно неразложимых, а через $\operatorname{Sim}(V)$ - класс простых алгебр из $V$. Через $\mathrm{FG}(V)$ обозначается класс конечно порожденных алгебр из класса $V$.

Конгруэнция называется неразложимой, если она не представима как пересечение конечного числа отличных от нее конгруэнций, и вполне неразложимой, если она не представима 
как пересечение отличных от нее конгруэнций. Отметим, что алгебра $\mathbf{A}$ финитно неразложима тогда и только тогда, когда $\mathrm{id}_{\mathbf{A}}$ неразложима; невырожденная алгебра $\mathbf{A}$ подпрямо неразложима тогда и только тогда, когда ее нулевая конгруэнция id $\mathbf{A}$ вполне неразложима.

Легко доказывается

ЛЕмма 4.2. Для любой собственной конгруэниии Ф на алгебре $\mathbf{A}$ :

- факторалгебра $\mathbf{A} / \Phi$ финитно неразложима, если и только если Ф неразложима;

- $\mathbf{A} / \Phi$ подпрямо неразложима, если и только если $\Phi$ вполне неразложима.

Лемма 4.3. Пусть $a, b \in \mathbf{A}, a \neq b$. Тогда существует гомоморфизм $h$ из $\mathbf{A}$ на подходящую подпрямо неразложимую алгебру $\mathbf{B}$ такой, что $h(a) \neq h(b)$.

ДокАЗАтельство. Семейство всех конгруэнций, не содержащих пару $(a, b)$, непусто и удовлетворяет условиям леммы Цорна, а значит, имеет максимальный элемент, который и будет вполне неразложимой конгруэнцией.

Для многообразий со свойством продолжаемости конгруэнций справедлива

Лемма 4.4. Пусть многообразие $V$ имеет $\mathrm{CEP}, \mathbf{A}, \mathbf{B} \in V, \mathbf{A}$ - подалгебра алгебры $\mathbf{B}$, $\Phi$ - конгруэниия на А. Если $\Phi$ неразложима, то существует неразложимая конгруэниия $\Psi$ на $\mathbf{B}$ такая, что $\Psi \cap \mathbf{A}^{2}=\Phi$.

ДоказАтельство. Рассмотрим множество

$$
\Sigma=\left\{\Psi \mid \Psi-\text { конгруэнция на } \mathbf{B} \text { и } \Psi \cap \mathbf{A}^{2}=\Phi\right\} .
$$

По СЕР множество $\Sigma$ непусто. Кроме того, $\Sigma$ частично упорядочено по включению и объединение любой цепи элементов из $\Sigma$ снова принадлежит $\Sigma$. По лемме Цорна $\Sigma$ содержит максимальный элемент $\Psi_{0}$. Нетрудно показать, что $\Psi_{0}$ неразложима.

4.1. Амальгамируемость и ограниченная амальгамируемость. Рассмотрим условия сведения для свойств амальгамируемости AP и ограниченной амальгамируемости RAP. Имеет место

Лемма 4.5 [23]. Для любого многообразия $V$ следующие условия эквивалентны:

(1) $V$ амальгамируемо;

(2) для любых $\mathbf{A}, \mathbf{B}, \mathbf{C} \in V$ maких, что $\mathbf{A}$ является общей подалгеброй алгебр $\mathbf{B} u \mathbf{C}, u$ для любых $c, c^{\prime} \in \mathbf{C}$, если $c \neq c^{\prime}$, то существуют подпрямо неразложимая алгебра $\mathbf{D}$ из $V$ и гомоморфизмы $g: \mathbf{B} \rightarrow \mathbf{D}, h: \mathbf{C} \rightarrow \mathbf{D}$ такие, что $g(x)=h(x)$ для всех $x \in \mathbf{A} u$ $h(c) \neq h\left(c^{\prime}\right)$.

Следующая теорема доказана в [23] для свойства амальгамируемости.

ТЕОрема 4.6. Пусть многообразие $V$ имеет CEP и, кроме того, класс $\mathrm{SI}(V)$ замкнут относительно взятия подалгебр. Если $\mathrm{SI}(V)$ имеет $\mathrm{AP}$, то $V$ имеет $\mathrm{AP}$.

По аналогии можно доказать, что справедлива

Теорема 4.7. Пусть многообразие $V$ имеет СЕР и, кроме того, подалгебры подпрямо неразложимых алгебр из $V$ финитно неразложимы. Тогда если класс $\mathrm{FI}(V)$ финитно неразложимых алгебр из $V$ имеет $\mathrm{AP}$, то $V$ имеет AP.

Мы отметим, что условия теоремы 4.7 выполняются, например, в случае модальных алгебр и импликативных решеток в то время, как условие замкнутости класса $\mathrm{SI}(V)$ относительно подалгебр не выполняется.

В общем случае из амальгамируемости многообразия не следует амальгамируемость классов подпрямо неразложимых или финитно неразложимых алгебр: это не выполняется даже для модальных алгебр, хотя и выполняется для гейтинговых алгебр и импликативных решеток [15]. В этой связи оказывается полезным следующее свойство. 
$\mathrm{APF}$ : для любых алгебр $\mathbf{A}, \mathbf{B}, \mathbf{C} \in V$ таких, что $\mathbf{B}, \mathbf{C}$ - конечно порожденные и финитно неразложимые и $\mathbf{A}$ является общей подалгеброй алгебр $\mathbf{B}$ и $\mathbf{C}$, и для любой пары $c_{1}, c_{2}$ различных элементов из $\mathbf{C}$ существуют конечно порожденная подпрямо неразложимая $\mathbf{D}$ из $V$ и гомоморфизмы $\delta: \mathbf{B} \rightarrow \mathbf{D}$, $\varepsilon: \mathbf{C} \rightarrow \mathbf{D}$ такие, что $\delta(x)=\varepsilon(x)$ для всех $x \in \mathbf{A}$ и $\varepsilon\left(c_{1}\right) \neq \varepsilon\left(c_{2}\right)$.

Очевидно, в многообразиях $\mathrm{APF}$ равносильно свойству

$\mathrm{APF}^{\prime}$ : для любых конечно порожденных финитно неразложимых алгебр $\mathbf{B}, \mathbf{C} \in$ $V$, для любых мономорфизмов $\beta$ и $\gamma$ алгебры $\mathbf{A}$ в $\mathbf{B}$ и $\mathbf{C}$ соответственно и для любой пары $c_{1}, c_{2}$ различных элементов из $\mathbf{C}$ существуют конечно порожденная подпрямо неразложимая $\mathbf{D}$ из $V$ и гомоморфизмы $\delta: \mathbf{B} \rightarrow \mathbf{D}$, $\varepsilon: \mathbf{C} \rightarrow \mathbf{D}$ такие, что $\delta \beta(x)=\varepsilon \gamma(x)$ для всех $x \in \mathbf{A}$ и $\varepsilon\left(c_{1}\right) \neq \varepsilon\left(c_{2}\right)$.

ТЕОРема 4.8. Пусть многообразие $V$ обладает свойством СЕР $и$, кроме того, подалгебрь подпрямо неразложимых алгебр финитно неразложимы. Тогда $V$ амальгамируемо, если и только если $V$ имеет APF.

ДокАЗАтЕЛьство. В силу леммы 4.3 ясно, что АРF следует из АР. Пусть $V$ имеет APF. Докажем, что $V$ удовлетворяет условию (2) леммы 4.5. Пусть $\mathbf{A}, \mathbf{B}, \mathbf{C} \in V, \mathbf{A}$ является общей подалгеброй алгебр $\mathbf{B}$ и $\mathbf{C}, c, c^{\prime} \in \mathbf{C}, c \neq c^{\prime}$. По лемме 4.3 существует вполне неразложимая конгруэнция $\Psi$ на $\mathbf{C}$ такая, что $\left(c, c^{\prime}\right) \notin \Psi$. При этом алгебра $\mathbf{C}_{1}=\mathbf{C} / \Psi$ подпрямо неразложима и является гомоморфным образом алгебры $\mathbf{C}$ относительно гомоморфизма $f(z)=z / \Psi$. Пусть $\mathbf{A}_{1}$ - образ алгебры $\mathbf{A}$ относительно $f$. По условию теоремы алгебра $\mathbf{A}_{1}$ финитно неразложима. Это означает, что конгруэнция $\Psi_{0}=\Psi \cap \mathbf{A}^{2}$ алгебры $\mathbf{A}$ неразложима. По лемме 4.4 существует неразложимая конгруэнция $\Phi$ на $\mathbf{B}$ такая, что $\Psi_{0}=\Phi \cap \mathbf{A}^{2}$. Обозначим $\mathbf{B}{ }_{1}=\mathbf{B} / \Phi$. Тогда $\mathbf{B}_{1}$ финитно неразложима и отображение $\beta: \mathbf{A}_{1} \rightarrow \mathbf{B}_{1}$, где $\beta(x / \Psi)=x / \Phi$ для $x \in \mathbf{A}$, является мономорфизмом. По $\mathrm{APF}^{\prime}$ существуют конечно порожденная подпрямо неразложимая $\mathbf{D}$ из $V$ и гомоморфизмы $\delta: \mathbf{B}_{\mathbf{1}} \rightarrow \mathbf{D}, \varepsilon: \mathbf{C}_{\mathbf{1}} \rightarrow \mathbf{D}$ такие, что $\delta \beta f(x)=\varepsilon f(x)$ для всех $x \in \mathbf{A}$ и $\varepsilon f(c) \neq \varepsilon f\left(c^{\prime}\right)$. Тогда $g(y)=\delta(y / \Phi)$ для $y \in \mathbf{B}$ и $h(z)=\varepsilon f(z)$ для $z \in \mathbf{C}-$ требуемые гомоморфизмы.

Перейдем к ограниченной амальгамируемости. Следующие утверждения доказаны в [12].

ПрЕДЛОЖЕНИЕ 4.9. Для любого многообразия $V$ следующие условия эквивалентны:

(1) $V$ uмеет RAP;

(2) для любых мономорфизмов $\beta: \mathbf{A} \rightarrow \mathbf{B} u \gamma: \mathbf{A} \rightarrow \mathbf{C}$ алгебр из $V$ существуют алгебра $\mathbf{D}$ из $V u$ гомоморфизмы $\delta: \mathbf{B} \rightarrow \mathbf{D}, \varepsilon: \mathbf{C} \rightarrow \mathbf{D}$ такие, что $\delta \beta=\varepsilon \gamma$ u $\delta \beta$ является мономорфизмом;

(3) для любых $\mathbf{A}, \mathbf{B}, \mathbf{C} \in V$ maких, что $\mathbf{A}$ является общей подалгеброй алгебр $\mathbf{B} u \mathbf{C}, u$ для любых $а, a^{\prime} \in \mathbf{A}$, если $a \neq a^{\prime}$, то существуют алгебра $\mathbf{D}$ из $V$ и гомоморбизмы $\delta: \mathbf{B} \rightarrow \mathbf{D}, \varepsilon: \mathbf{C} \rightarrow \mathbf{D}$ maкие, что $\delta(x)=\varepsilon(x)$ для всех $x \in \mathbf{A} u \delta(a) \neq \delta\left(a^{\prime}\right)$.

ПреДЛОЖЕНИЕ 4.10. Пусть многообразие $V$ имеет СЕР. Тогда следующие условия эквивалентны:

- $V$ uмeеm RAP.

- для любых мономорфизмов $\beta: \mathbf{A} \rightarrow \mathbf{B} u \gamma: \mathbf{A} \rightarrow \mathbf{C}$ подпрямо неразложсимъх алгебр из $V$ существуют подпрямо неразложимая алгебра $\mathbf{D}$ из $V$ и гомоморфизмы $\delta: \mathbf{B} \rightarrow \mathbf{D}$, $\epsilon: \mathbf{C} \rightarrow \mathbf{D}$ такие, что $\delta \beta=\epsilon \gamma$ и $\delta \beta$ есть мономорфизм.

Учитывая теорему $3.5,(2)$, можно в предложении 4.10 ограничиться конечно порожденными алгебрами $\mathbf{A}, \mathbf{B}, \mathbf{C}, \mathbf{D}$. Из предложения 4.10 сразу вытекает

Tеорема 4.11. Пусть многообразие $V$ имеет CEP. Тогда $V$ имеет RAP, если и только если класс $\mathrm{FGSI}(V)$ конечно порожденных подпрямо неразложимых алгебр из $V$ имеет RAP. 
4.2. Слабая амальгамируемость и простые алгебры. Слабое интерполяционное свойство и слабая амальгамируемость в многообразиях ограниченных алгебр изучались в [14]. Приведем основные результаты этой статьи.

ТЕОРема 4.12. Пусть $V$ - многообразие ограниченнъх алгебр. Тогда

(1) если $V$ имеет WIP, то оно имеет WAP;

(2) если $V$ имеет CEP и $\mathrm{FG}(V)$ имеет WAP, mо $V$ uмеет WIP.

Непосредственно из теоремы вытекает

СЛЕДСТВИЕ 4.13. Для любого многообразия $V$ ограниченных алгебр со свойством СЕР следующие условия эквивалентны:

(1) $V$ uмеет WIP;

(2) $V$ uмеет WAP;

(3) $\mathrm{FG}(V)$ uмeеm WAP.

Для многообразий модальных алгебр эквивалентность WIP и WAP следует из [28, теорема 2.3].

Напомним, что невырожденная алгебра называется простой, если она имеет точно две конгруэнции. Легко видеть, что в ограниченной алгебре конгруэнция $\Theta$ является собственной тогда и только тогда, когда $(\perp, \top) \notin \Theta$. Для любой собственной конгруэнции $\Theta$ ограниченной алгебры $\mathbf{A}$ существует максимальная конгруэнция $\Theta^{\prime} \supseteq \Theta$; алгебра $\mathbf{A} / \Theta$ является простой тогда и только тогда, когда $\Theta$ - максимальная конгруэнция на $\mathbf{A}$.

Легко видеть, что справедлива следующая

ЛЕмма 4.14. Если многообразие $V$ имеет СЕР, то невырожденная подалгебра простой алгебры из $V$ является простой алгеброй.

ТЕОРемА 4.15 [14]. Пусть многообразие $V$ ограниченных алгебр имеет СЕР. Тогда следующие условия эквивалентны:

(1) $V$ слабо амальгамируемо;

(2) класс простых алгебр из $V$ амальгамируем;

(3) класс конечно порожденных простых алгебр из $V$ амальгамируем.

В [35] описаны все конечно порожденные, простые транзитивные модальные алгебры. Это позволило доказать разрешимость слабой амальгамируемости в многообразиях транзитивных модальных алгебр.

В [23] доказано, что существуют точно четыре нетривиальных амальгамируемых многообразия дистрибутивных решеток с псевдодополнениями. С другой стороны, имеется лишь одна простая дистрибутивная решетка с псевдодополнениями - это двухэлементная булева алгебра. Поэтому все многообразия дистрибутивных решеток с псевдодополнениями имеют WAP и WIP.

Аналогично, каждый из классов простых гейтинговых, диагонализируемых и гжегорчиковых алгебр состоит из одной двухэлементной алгебры. Поэтому все подмногообразия гейтинговых, диагонализируемых и гжегорчиковых алгебр имеют WAP и WIP [28].

С другой стороны, в многобразиях эпистемических алгебр слабая амальгамируемость равносильна сверхамальгамируемости [28]. Все амальгамируемые многообразия эпистемических алгебр были описаны в [27], [42]. Можно показать, что простые эпистемические алгебры - это в точности модальные алгебры $\mathbf{A}=(A, \wedge, \neg, \top, \square)$, где $\square \top=\top, \square x=\perp$ при $x \neq \top$. Кроме тривиального многообразия и многообразия всех эпистемических алгебр, амальгамируемыми являются многообразия, порожденные двухэлементной или четырехэлементной алгеброй указанного вида. 
4.3. Сверхамальгамируемость и свойство отделимости. В формулировке свойства сверхамальгамируемости предполагается, что все алгебры частично упорядочены. Мы ограничимся рассмотрением классов алгебр, основанных на нижних полурешетках. То есть предполагаем, что в многообразиях выразима бинарная операция $\wedge$ взятия точной нижней грани.

Неизвестно, как в общем случае свести свойство сверхамальгамируемости к финитно неразложимым алгебрам. В случае модальных или гейтинговых алгебр такое сведение возможно (см. теорему 4.1) и играет большую роль в изучении сверхамальгамируемости. По теореме 3.5 сверхамальгамируемость равносильна конъюнкции свойств AP и WSupAP. Теорема 4.8 дает достаточные условия для сведения свойства АР.

Теперь рассмотрим вопрос о“финитизации свойств IIP и WSupAP. Когда свойство WSupAP можно свести к рассмотрению финитно неразложимых алгебр?

Определим свойство

FWSupAP : для любой алгебры $\mathbf{A}$, любых финитно неразложимых и конечно порожденных $\mathbf{B}, \mathbf{C} \in V$, если $\mathbf{A}$ является общей подалгеброй алгебр $\mathbf{B}$ и $\mathbf{C}$, элементы $b \in \mathbf{B}, c \in \mathbf{C}$ удовлетворяют условию $\neg(\exists a \in \mathbf{A})(b \leqslant a \& a \leqslant c)$, то существуют подпрямо неразложимая $\mathbf{D} \in V$ и гомоморфизмы $g: \mathbf{B} \rightarrow \mathbf{D}$, $h: \mathbf{C} \rightarrow \mathbf{D}$ такие, что $g(a)=h(a)$ для всех $a \in \mathbf{A}$ и $g(b) \nless h(c)$.

Нетрудно понять, что в многообразиях свойство FWSupAP равносильно следующему свойству.

FWSupAP' $^{\prime}$ для любой алгебры $\mathbf{A}$, любых финитно неразложимых и конечно порожденных $\mathbf{B}, \mathbf{C} \in V$, любых мономорфизмов $\beta: \mathbf{A} \rightarrow \mathbf{B}$ и $\gamma: \mathbf{A} \rightarrow \mathbf{C}$ и любых элементов $b \in \mathbf{B}, c \in \mathbf{C}$ таких, что $\neg(\exists a \in \mathbf{A})(b \leqslant \beta(a) \& \gamma(a) \leqslant c)$, существуют подпрямо неразложимая $\mathbf{D} \in V$ и гомоморфизмы $g: \mathbf{B} \rightarrow \mathbf{D}$, $h: \mathbf{C} \rightarrow \mathbf{D}$ такие, что $g \beta=h \gamma$ и $g(b) \nless h(c)$.

Мы сформулируем свойство отделимости SEP, которое справедливо для модальных и гейтинговых алгебр и существенно использовалось при исследовании амальгамируемости в многообразиях этих алгебр.

$\mathrm{SEP}$ : пусть $\mathbf{A}$ является обшей подалгеброй алгебр $\mathbf{B}$ и $\mathbf{C}$ из $V, b \in \mathbf{B}, c \in \mathbf{C}$ и $\neg(\exists x \in \mathbf{A})(b \leqslant x \& x \leqslant c)$. Тогда существуют неразложимые конгруэнции $\Phi$ на $\mathbf{B}$ и $\Psi$ на $\mathbf{C}$ такие, что $\Phi \cap \mathbf{A}^{2}=\Psi \cap \mathbf{A}^{2}$ и $\neg(\exists a \in \mathbf{A})(b / \Phi \leqslant a / \Phi \& a / \Psi \leqslant$ $c / \Psi)$.

Ясно, что в случае полурешеток условие $\neg(\exists a \in \mathbf{A})(b / \Phi \leqslant a / \Phi \& a / \Psi \leqslant c / \Psi)$ равносильно условию $\neg(\exists a \in \mathbf{A})((b, b \wedge a) \in \Phi \&(a, a \wedge c) \in \Psi)$.

Теорема 4.16. Для любого многообразия $V$ полурешеточно упорядоченных алгебр, обладающего свойством отделимости SEP, следующие условия эквивалентны:

(1) $V$ имеет IIP;

(2) $V$ uмеет WSupAP;

(3) $V$ имеет FWSupAP.

ДокАЗАТЕЛЬСтво. По теореме 3.7 IIP и WSupAP равносильны. Мы докажем

$$
\text { WSupAP } \Longrightarrow \text { FWSupAP } \quad \text { и } \quad \text { FWSupAP } \Longrightarrow \text { IIP. }
$$

$(2) \Rightarrow(3)$. Пусть $V$ имеет WSupAP. Докажем, что $V$ имеет FWSupAP. Пусть даны алгебpa $\mathbf{A}$, финитно неразложимые и конечно порожденные $\mathbf{B}, \mathbf{C} \in V$ такие, что $\mathbf{A}$ является общей подалгеброй алгебр $\mathbf{B}$ и $\mathbf{C}$, и элементы $b \in \mathbf{B}, c \in \mathbf{C}$ удовлетворяют условию $\neg(\exists a \in \mathbf{A})(b \leqslant$ $a \& a \leqslant c)$. По WSupAP существуют $\mathbf{D}$ из $V$ и гомоморфизмы $g: \mathbf{B} \rightarrow \mathbf{D}$ и $h: \mathbf{C} \rightarrow \mathbf{D}$ такие, что $g(x)=h(x)$ для всех $x \in \mathbf{A}$ и, кроме того,

$$
g(x) \leqslant h(y) \Longleftrightarrow(\exists z \in \mathbf{A})(x \leqslant z \text { и } z \leqslant y) .
$$


Ясно, что можно считать алгебру $\mathbf{D}$ конечно порожденной. Кроме того, имеем $g(b) \not k h(c)$, т.е. $g(b) \wedge h(c) \neq g(b)$. По лемме 4.3 существует гомоморфизм $f$ из $\mathbf{D}$ на подходящую подпрямо неразложимую алгебру $\mathbf{D}^{\prime}$ такой, что $f(g(b)) \wedge f(h(c)) \neq f(g(b))$, что равносильно $f(g(b)) \not$ $f(h(c))$. Тогда $\mathbf{D}^{\prime}$ - искомая алгебра, которая требуется в FWSupAP.

$(3) \Rightarrow(1)$. Допустим, для доказательства от противного, что $V$ имеет свойство FWSupAP', равносильное FWSupAp, но не имеет IIP. Существуют $\Gamma(\mathbf{x}, \mathbf{y})$ и $\Delta(\mathbf{x}, \mathbf{z})$, удовлетворяющие условиям:

(1) $\Gamma^{\prime}(\mathbf{x})=\Delta^{\prime}(\mathbf{x})$, где

$$
\Gamma^{\prime}(\mathbf{x})=\left\{\alpha(\mathbf{x}) \mid \Gamma(\mathbf{x}, \mathbf{y}) \models_{V} \alpha(\mathbf{x})\right\}, \quad \Delta^{\prime}(\mathbf{x})=\left\{\alpha(\mathbf{x}) \mid \Delta(\mathbf{x}, \mathbf{z}) \models_{V} \alpha(\mathbf{x})\right\} ;
$$

(2) $\Gamma(\mathbf{x}, \mathbf{y}), \Delta(\mathbf{x}, \mathbf{z}) \models_{V} u(\mathbf{x}, \mathbf{y}) \leqslant v(\mathbf{x}, \mathbf{z})$;

(3) не существует терма $t(\mathbf{x})$ такого, что $\Gamma(\mathbf{x}, \mathbf{y}) \models_{V} u(\mathbf{x}, \mathbf{y}) \leqslant t(\mathbf{x})$ и $\Delta(\mathbf{x}, \mathbf{z}) \models_{V} t(\mathbf{x}) \leqslant$ $v(\mathbf{x}, \mathbf{z})$.

Без ограничения общности можно считать. что списки $\mathbf{x}, \mathbf{y}, \mathbf{z}$ конечны. На множестве термов $F(\mathbf{x}, \mathbf{y})$ определяем конгруэнцию

$$
\tau(\mathbf{x}, \mathbf{y}) \approx_{1} \tau^{\prime}(\mathbf{x}, \mathbf{y}) \Longleftrightarrow \Gamma(\mathbf{x}, \mathbf{y}) \models_{V} \tau(\mathbf{x}, \mathbf{y})=\tau^{\prime}(\mathbf{x}, \mathbf{y}),
$$

а множестве $F(\mathbf{x}, \mathbf{z})$ - конгруэнцию

$$
\tau(\mathbf{x}, \mathbf{z}) \approx_{2} \tau^{\prime}(\mathbf{x}, \mathbf{z}) \Longleftrightarrow \Delta(\mathbf{x}, \mathbf{z}) \models_{V} \tau(\mathbf{x}, \mathbf{z})=\tau^{\prime}(\mathbf{x}, \mathbf{z}) .
$$

Положим $\mathbf{B}=F(\mathbf{x}, \mathbf{y}) / \approx_{1}, \mathbf{C}^{\prime}=F(\mathbf{x}, \mathbf{z}) / \approx_{2}$.

Пусть $\mathbf{A}$ - подалгебра алгебры $\mathbf{B}$, порожденная множеством $\mathbf{x} / \approx_{1}$. Из условия $\Gamma^{\prime}(\mathbf{x})=$ $\Delta^{\prime}(\mathbf{x})$ следует, что $\tau(\mathbf{x}) \approx_{1} \tau^{\prime}(\mathbf{x}) \Longleftrightarrow \tau(\mathbf{x}) \approx_{2} \tau^{\prime}(\mathbf{x})$ для любых термов $\tau(\mathbf{x}), \tau^{\prime}(\mathbf{x})$, а значит, отображение $\varphi\left(\tau(\mathbf{x}) / \approx_{1}\right)=\left(\tau(\mathbf{x}) / \approx_{2}\right)$ является мономорфизмом из $\mathbf{A}$ в $\mathbf{C}^{\prime}$. Отображение $\varphi^{-1}$ можно продолжить до изоморфизма $\psi$ из $\mathbf{C}^{\prime}$ на подходящую алгебру $\mathbf{C}$ такую, что $\mathbf{A}$ является подалгеброй алгебры $\mathbf{C}$.

Положим $b=u(\mathbf{x}, \mathbf{y}) / \approx_{1}, c=\varphi^{-1}\left(v(\mathbf{x}, \mathbf{z}) / \approx_{2}\right)$. По условию (3) не существует элемента $a \in \mathbf{A}$ такого, что $b \leqslant a$ и $a \leqslant c$.

Применяем свойство отделимости. Существуют неразложимые конгруэнции $\Phi$ на $\mathbf{B}$ и $\Psi$ на $\mathbf{C}$ такие, что $\Phi \cap \mathbf{A}^{2}=\Psi \cap \mathbf{A}^{2}$ и $\neg(\exists a \in \mathbf{A})(b / \Phi \leqslant a / \Phi \& a / \Psi \leqslant c / \Psi)$. Обозначим $\mathbf{B}_{1}=\mathbf{B} / \Phi$, $\mathbf{C}_{1}=\mathbf{C} / \Psi$. Тогда $\mathbf{B}_{1}$ и $\mathbf{C}_{1}$ финитно неразложимы и образ $\mathbf{A}_{1}$ подалгебры $\mathbf{A}$ в $\mathbf{B}_{1}$ изоморфно

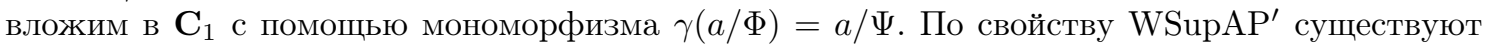
подпрямо неразложимая алгебра $\mathbf{D} \in V$ и гомоморфизмы $g: \mathbf{B}_{1} \rightarrow \mathbf{D}, h: \mathbf{C}_{1} \rightarrow \mathbf{D}$ такие, что $g(b / \Phi) \nless{ }^{\prime}(c / \Psi)$ и $g(a / \Phi)=h \gamma(a / \Phi)$ для всех $a \in \mathbf{A}$.

Строим означивание $v$ формул от переменных $\mathbf{x}, \mathbf{y}, \mathbf{z}$ в алгебре $\mathbf{D}$, полагая

$$
v(y)=g\left(\left(y / \approx_{1}\right) / \Phi\right) \quad \text { для } y \in(\mathbf{x} \cup \mathbf{y}), \quad v(z)=h\left(\left(z / \approx_{2}\right) / \Psi\right) \quad \text { для } z \in \mathbf{z} .
$$

Тогда, учитывая, что $h\left(\left(x / \approx_{2}\right) / \Psi\right)=v(x)$ для $x \in \mathbf{x}$, получаем

$$
\mathbf{D} \models \Gamma(\mathbf{x}, \mathbf{y})[v], \quad \mathbf{D} \models \Delta(\mathbf{x}, \mathbf{z})[v] .
$$

Из условия $(2)$ заключаем, что $\mathbf{D} \models(u(\mathbf{x}, \mathbf{y}) \leqslant v(\mathbf{x}, \mathbf{z}))[v]$, т.е. $g(b / \Phi) \leqslant h(c / \Psi)-$ противоречие.

4.4. Свойства Бета и финитно неразложимые алгебры. В общем случае свойство Бета не сводится к финитно неразложимым алгебрам. Например, многообразие ограниченных дистрибутивных решеток не обладает свойством Бета (см., например, [21]), хотя класс финитно неразложимых алгебр из этого многообразия состоит лишь из двухэлементных решеток, не имеющих собственных подалгебр, и, очевидно, обладает свойством ES*, эквивалентным BР.

Для сведения свойств Бета нам достаточно следующего свойства отделимости SEP1, которое вытекает из SEP: 
SEP1 : пусть А является подалгеброй алгебры $\mathbf{B}$ из $V, b \in \mathbf{B}-\mathbf{A}$; тогда существуют неразложимые конгруэнции $\Phi$ и $\Psi$ на $\mathbf{B}$ такие, что $\Phi \cap \mathbf{A}^{2}=\Psi \cap \mathbf{A}^{2}$ и $\neg(\exists a \in \mathbf{A})((a, b) \in \Phi \&(a, b) \in \Psi)$.

Легко вывести SEP1 из SEP: достаточно положить в $\mathrm{SEP} \mathbf{C}=\mathbf{B}$ и $c=b$.

ТеОрема 4.17. Пусть многообразие V имеет SEP1. Тогда следующие условия эквивалентны:

(1) V имеет свойство Бета ВР;

(2) для любой конечно порожденной, финитно неразложимой алгебры $\mathbf{B} \in V u$ любого

$b \in \mathbf{B}-\mathbf{A}$ такого, что $\mathbf{A} \cup\{b\}$ порождает $\mathbf{A}$, существуют подпрямо неразложимая алгебра $\mathbf{C} \in V$ u гомоморфизмы $g: \mathbf{B} \rightarrow \mathbf{C} u h: \mathbf{B} \rightarrow \mathbf{C}$ maкие, что $g(b) \neq h(b) u$ $g(x)=h(x)$ для всех $x \in \mathbf{A}$.

ДоказАтельство. (1) $\Rightarrow(2)$. Следует из эквивалентности ВР и $\mathrm{ES}^{*}$ (теорема 3.12 ).

$(2) \Rightarrow(1)$. Допустим, что $V$ не обладает свойством ВР, и найдем контрпример для (2).

Существует множество равенств $\Gamma(\mathbf{p}, x)$ такое, что

$$
\Gamma(\mathbf{p}, x), \Gamma(\mathbf{p}, y) \models_{V} x=y, \quad \neg \exists t(\mathbf{p})\left(\Gamma(\mathbf{p}, x) \models_{V} x=t(\mathbf{p})\right) .
$$

Очевидно, список р можно считать конечным. Берем следующую конгруэнцию на алгебре термов $F(\mathbf{p}, x)$ :

$$
u(\mathbf{p}, x) \approx v(\mathbf{p}, x) \Longleftrightarrow \Gamma(\mathbf{p}, x) \models_{V} u(\mathbf{p}, x)=v(\mathbf{p}, x) .
$$

Обозначим через $\mathbf{B}$ факторалгебру алгебры $F(\mathbf{p}, x)$ по этой конгруэнции, а через $\mathbf{A}-$ подалгебру алгебры $\mathbf{B}$, состоящую из образов термов из $F(\mathbf{p})$. Для любой переменной $z \in \mathbf{p} \cup\{x\}$ обозначаем $z^{\prime}=z / \approx$. Тогда $x^{\prime} \in \mathbf{B}-\mathbf{A}$. По свойству SEP1 существуют неразложимые конгруэнции $\Phi$ и $\Psi$ на $\mathbf{B}$ такие, что $\Phi \cap \mathbf{A}^{2}=\Psi \cap \mathbf{A}^{2}$ и $\neg(\exists a \in \mathbf{A})\left(\left(x^{\prime}, a\right) \in \Phi\right.$ и $\left.\left(x^{\prime}, a\right) \in \Psi\right)$.

Обозначим $\mathbf{B}_{1}=\mathbf{B} / \Phi, b=x^{\prime} / \Phi, \mathbf{A}_{1}=\{a / \Phi \mid a \in \mathbf{A}\}$. Покажем, что $b \in \mathbf{B}_{1}-\mathbf{A}_{1}$.

Допустим (для доказательства от противного), что $b \in \mathbf{A}_{1}$, т.е. существует $a \in \mathbf{A}$ такой, что $x^{\prime} / \Phi=a / \Phi$. Тогда $\left(x^{\prime}, a\right) \in \Phi$. Кроме того, для любого равенства $\gamma(\mathbf{p}, x) \in \Gamma(\mathbf{p}, x)$ имеем $\mathbf{B} \models \gamma\left(\mathbf{p}^{\prime}, x^{\prime}\right)$, поэтому $\gamma\left(\mathbf{p}^{\prime}, x^{\prime}\right) \in \Phi$. Из $\left(x^{\prime}, a\right) \in \Phi$ получаем $\gamma\left(\mathbf{p}^{\prime}, a\right) \in \Phi$, а значит, $\gamma\left(\mathbf{p}^{\prime}, a\right) \in \Psi$. Таким образом, $\Gamma\left(\mathbf{p}^{\prime}, a\right) \subseteq \Psi$.

Из условия $\mathbf{B}=\Gamma\left(\mathbf{p}^{\prime}, x^{\prime}\right)$ заключаем, что $\Gamma\left(\mathbf{p}^{\prime}, x^{\prime}\right) \subseteq \Psi$. Учитывая $\Gamma\left(\mathbf{p}^{\prime}, a\right) \subseteq \Psi$ и $\Gamma(\mathbf{p}, x), \Gamma(\mathbf{p}, y) \models_{V} x=y$, получаем $\left(x^{\prime}, a\right) \in \Psi$, что противоречит условию $\neg(\exists a \in \mathbf{A})\left(\left(x^{\prime}, a\right) \in\right.$ $\Phi$ и $\left.\left(x^{\prime}, a\right) \in \Psi\right)$. Следовательно, $b \in \mathbf{B}_{1}-\mathbf{A}_{1}$.

Поскольку конгруэнция $\Phi$ является неразложимой, алгебра $\mathbf{B}_{1}$ финитно неразложима. Кроме того, $\mathbf{B}_{1}$ - конечно порожденная и $\mathbf{A}_{1} \cup\{b\}$ порождает $\mathbf{B}_{1}$.

Допустим, что существуют подпрямо неразложимая $\mathbf{D} \in V$ и два гомоморфизма $h$ и $g$ из $\mathbf{B}_{1}$ в $\mathbf{D}$ такие, что $h(a)=g(a)$ для всех $a \in \mathbf{A}_{1}$ и $h(b) \neq g(b)$. Рассмотрим следующее означивание $v$ в алгебре $\mathbf{D}$ :

$$
\begin{gathered}
v(p)=g\left(p^{\prime} / \Phi\right)=h\left(p^{\prime} / \Phi\right) \quad \text { для любой переменной } \quad p \in \mathbf{p}, \\
v(x)=g(b), \quad v(y)=h(b) .
\end{gathered}
$$

Тогда $\mathbf{D} \models \Gamma(\mathbf{p}, x)[v], \mathbf{D} \models \Gamma(\mathbf{p}, y)[v]$, однако $\mathbf{D} \not \models(x=y)[v]$, что противоречит $\Gamma(\mathbf{p}, x), \Gamma(\mathbf{p}, y) \models_{V} x=y$. Теорема доказана.

Для проективного свойства Бета недостаточно, чтобы класс конечно порожденных финитно неразложимых алгебр из $V$ имел свойство SES даже в случае, когда $V$ имеет свойство SEP1 или даже более сильное свойство SEP. Простой контрпример был найден в [26] в классе гейтинговых алгебр. Там показано, что многообразие, порожденное 5-элементной линейно упорядоченной гейтинговой алгеброй, не имеет свойства SES. C другой стороны, для любой линейно упорядоченной гейтинговой алгебры $\mathbf{B}$ и любой ее подалгебры легко найти требуемые гомоморфизмы из В в В. Тем не менее, при условии SEP1 сведение существует. Следующая 
теорема является аналогом теоремы 3.8 из [26], доказанной для многообразий модальных алгебр.

TЕОРема 4.18. Пусть многообразие $V$ имеет SEP1. Тогда следующие условия эквивалентны:

(1) $V$ имеет проективное свойство Бета РВР;

(2) для любых конечно порожденных финитно неразложимых алгебр $\mathbf{B} u \mathbf{C}$, имеющих общую подалгебру $\mathbf{A}, u$ любых элементов $b \in \mathbf{B}, c \in \mathbf{C} \operatorname{ma\kappa ux,~что~} \neg(\exists a \in \mathbf{A})(b=$ $a \& a=c)$, существуют подпрямо неразложимая алгебра $\mathbf{D} \in V$ u гомоморбизмы $g: \mathbf{B} \times \mathbf{C} \rightarrow \mathbf{D} u h: \mathbf{B} \times \mathbf{C} \rightarrow \mathbf{D}$ maкue, umo $g(b, c) \neq h(b, c)$ u $g(a, a)=h(a, a)$ для $8 c e x$ $a \in \mathbf{A}$.

ДокАЗАТЕЛЬСтво. Используем равносильность PBP и SES (теорема 3.12).

$(1) \Rightarrow(2)$. Пусть даны $\mathbf{B}, \mathbf{C}$ из $V$, имеющие общую подалгебру $\mathbf{A}$, и $b \in \mathbf{B}, c \in \mathbf{C}$ такие, что $\neg(\exists a \in \mathbf{A})(b=a \& a=c)$. Тогда алгебра $\mathbf{A}^{\prime}=\{(z, z) \mid z \in \mathbf{A}\}$ является подалгеброй алгебры $\mathbf{D}=\mathbf{B} \times \mathbf{C}$ и $(b, c) \in \mathbf{D}-\mathbf{A}^{\prime}$. По SES существуют алгебра $\mathbf{D}^{\prime}$ в $V(L)$ и требуемые гомоморфизмы. При этом можно взять алгебру $\mathbf{D}^{\prime}$ подпрямо неразложимой.

$(2) \Rightarrow(1)$. В силу равносильности PBP и SES достаточно доказать SES для конечно порожденных алгебр. Пусть $\mathbf{A}$ и $\mathbf{B}$ - конечно порожденные алгебры из $V, \mathbf{A}$ - подалгебра алгебры $\mathbf{B}$ и $b \in \mathbf{B}-\mathbf{A}$. Тогда по SEP1 существуют неразложимые конгуэнции $\Phi$ и $\Psi$ на $\mathbf{B}$ такие, что $\Phi \cap \mathbf{A}^{2}=\Psi \cap \mathbf{A}^{2}$ и $\neg(\exists a \in \mathbf{A})((a, b) \in \Phi \&(a, b) \in \Psi)$.

Тогда алгебры $\mathbf{B}_{1}=\mathbf{B} / \Phi$ и $\mathbf{B}_{2}=\mathbf{B} / \Psi$ финитно неразложимы. Заметим, что алгебра $\mathbf{A}_{0}=\{a / \Phi \mid a \in \mathbf{A}\}$ изоморфно вложима в $\mathbf{B}_{2}$ с помощью мономорфизма $i_{2}(a / \Phi)=a / \Psi$, причем $\neg\left(\exists z \in \mathbf{A}_{0}\right)(b / \Phi=z \& b / \Psi=z)$. Существует изоморфизм $\varphi$ алгебры $\mathbf{B}_{2}$ на подходящую алгебру $\mathbf{C}$ такую, что $\mathbf{A}_{0}$ является подалгеброй С. Положим $b_{1}=b / \Phi, c=\varphi(b / \Psi)$. По условию (2) существуют $\mathbf{D} \in V$ и гомоморфизмы $g: \mathbf{B}_{1} \times \mathbf{C} \rightarrow \mathbf{D}$ и $h: \mathbf{B}_{1} \times \mathbf{C} \rightarrow \mathbf{D}$ такие, что $g\left(b_{1}, c\right) \neq h\left(b_{1}, c\right)$ и $g(a, a)=h(a, a)$ для всех $a \in \mathbf{A}_{0}$. Для $x \in \mathbf{B}$ полагаем $\beta(x)=g(x / \Phi, \varphi(x / \Psi)), \gamma(x)=h(x / \Phi, \varphi(x / \Psi))$. Тогда $\beta$ и $\gamma-$ требуемые гомоморфизмы из $\mathbf{B}$ B D.

\section{5. Многообразия, порожденные конечными алгебрами}

Применим результаты предыдущих параграфов к табличным многообразиям. Многообразие алгебр конечной сигнатуры называется табличным, если оно порождается конечным числом конечных алгебр или, эквивалентно, одной конечной алгеброй. Укажем достаточные условия для разрешимости интерполяционных свойств, вариантов амальгамируемости и свойства Бета в табличных многообразиях алгебр.

Теоремы 3.5, 3.6 и 4.1 позволяют эффективно доказать или опровергнуть рассматриваемые свойства в табличных многообразиях модальных алгебр, импликативных решеток или гейтинговых алгебр. В случае указанных табличных многообразий можно за конечное число шагов проверить, удовлетворяет ли подкласс, состоящий из финитно неразложимых алгебр условиям (1)-(7) теоремы 4.1. С использованием известной теоремы Йонссона [43] доказывается

Теорема 5.1. Следуюшие свойства являются разрешимыми на классах табличных многообразий модалъных или гейтинговых алгебр, или решеток с относительными псевдодополнениями:

(1) амальгамируемость, сверхамальгамируемость, сильная амальгамируемость, ограниченная амальгамируемость, слабая амальгамируемость;

(2) сюргективность эпиморфизмов $\mathrm{ES}^{*}$ и сильная сюргективность эпиморфизмов SES;

(3) интерполяиионные свойства EIP, IPR, WIP, IIP;

(4) свойство Бета ВР и проективное свойство Бета РВР. 
Напомним, что все указанные в теореме многообразия обладают свойством продолжаемости конгруэнций, поэтому свойства $\mathrm{EIP}^{*}, \mathrm{IPR}^{*}$ и $\mathrm{WIP}^{*}$ равносильны $\mathrm{EIP}^{*}, \mathrm{IPR}^{*}$ и $\mathrm{WIP}^{*}$ соответственно.

Для проверки свойств, указанных в теореме 5.1, по данному конечному множеству алгебр, порождающих многообразие $V$, достаточно построить конечный класс финитно неразложимых алгебр, содержащий все с точностью до изоморфизма финитно неразложимые алгебры из $V$, а затем проверить требуемые в теореме 4.1 условия.

Существенную роль в доказательстве играет тот факт, что все рассматриваемые многообразия конгруэнц-дистрибутивны, т.е. все алгебры, имеют дистрибутивные решетки конгруэнций. Если конгруэнц-дистрибутивное многобразие порождается конечным числом конечных алгебр, то по теореме Йонссона [43] оно имеет лишь конечное число (с точностью до изоморфизма) финитно неразложимых алгебр.

ПРЕДЛОЖЕНИЕ 5.2. Пусть $V$ - конгруэни-дистрибутивное многообразие, порожденное конечным множеством $K$ конечных алгебр конечной сигнатуры. Тогда любая финитно неразложимая алгебра из $V$ конечна, и можно эффективно найти конечный класс $K_{0}$ конечных алгебр, состоящий из всех (с точностью до изоморбизма) финитно неразложимых алгебр из $V$.

ДокАЗАТЕЛЬСтво. Пусть многообразие $V$ порождается конечным множеством $K$ конечных модальных алгебр. Поскольку многообразие конгруэнц-дистрибутивно, по теореме Йонссона любая подпрямо неразложимая алгебра из $V$ входит в класс $\mathrm{HSP}_{\mathrm{U}}(K)[43]$, где H, $\mathrm{S}$ и $\mathrm{P}_{\mathrm{U}}$ - замыкания класса алгебр относительно взятия гомоморфных образов, подалгебр и ультрапроизведений соответственно. Можно показать что это же верно и для финитно неразложимых алгебр. Любое ультрапроизведение алгебр из $K$ конечно и изоморфно некоторой алгебре из $K$. Отсюда следует, что любая финитно неразложимая алгебра из $V$ изоморфна некоторой алгебре из класса $\mathrm{HS}(K)$, поэтому любая финитно неразложимая алгебра конечна. Кроме того, класс $\mathrm{HS}(K)$ является конечным с точностью до изоморфизма. Проверка свойства финитной неразложимости также является эффективной. Таким образом, получим конечный подкласс $K_{0}$, содержащий с точностью до изоморфизма все финитно неразложимые алгебры из $V$.

Используя критерии, найденные в предыдущих параграфах, установим результаты о разрешимости исследуемых в работе свойств в табличных многообразиях. Предполагаем, что табличное многообразие задается с помощью конечной алгебры, порождающей это многообразие. Обозначаем через $V(\mathbf{A})$ многообразие, порожденное алгеброй А. Учитывая предложение 5.2, мы ограничимся рассмотрением конгруэнц-дистрибутивных многообразий конечной сигннатуры.

ТеОРема 5.3. Пусть $V$ - конгруэни-дистрибутивное многообразие конечной сигнатуръ, обладающее свойством продолжсаемости конгруэниий СЕР. Тогда

(1) существует алгоритм, позволяющий для любой конечной алгебры $\mathbf{A} \in V$ определить, обладает ли многообразие $V(\mathbf{A})$ свойствами RAP и IPR;

(2) если подалгебры подпрямо неразложимых алгебр из $V$ бинитно неразложимы, то существует алгоритм, позволяющий для любой конечной алгебры $\mathbf{A} \in V$ определить, обладает ли многообразие $V(\mathbf{A})$ свойствами $\mathrm{AP} u \mathrm{EIP}$;

(3) если $V$ - многообразие ограниченных алгебр, то существует алгоритм, позволяющий для любой конечной алгебры $\mathbf{A} \in V$ определить, обладает ли многообразие $V(\mathbf{A})$ свойствами WAP $и$ WIP.

ДокАЗАТЕЛЬСтво. По теореме 3.5 свойство RAP равносильно IPR, AP равносильно ЕIP, a WAP эквивалентно WIP. По предложению 5.2 эффективно строится конечный класс, состоящий из всех с точностью до изоморфизма финитно неразложимых алгебр из $V(\mathbf{A})$. Далее используем теоремы $4.11,4.5$ и 4.15 . 
ТеОрема 5.4. Пусть $V$ - конгруэнц-дистрибутивное многообразие полурешеточно упорядоченных алгебр конечной сигнатуры, обладающее свойством отделимости SEP. Тогда существует алгоритм, позволяющий для любой конечной алгебры $\mathbf{A} \in V$ определить, обладает ли многообразие $V(\mathbf{A})$ свойствами IIP $u$ WSupAP.

ДокАЗАтельство. Следует из теоремы 3.7, предложения 5.2 и теоремы 4.16.

ТЕОРема 5.5. Пусть $V$ - конгруэни-дистрибутивное многообразие конечной сигнатуры, обладающее свойством отделимости SEP1. Тогда существует алгоритм, позволяющий для любой конечной алгебрь $\mathbf{A} \in V$ определить, обладает ли многообразие $V(\mathbf{A})$ свойствами $\mathrm{BP}, \mathrm{PBP}, \mathrm{ES}^{*}$ или SES.

ДокАЗАТЕЛьство. Следует из теоремы 3.12, предложения 5.2 и теорем 4.17 и 4.18 .

ТЕОРема 5.6. Пусть $V$ - конгруэни-дистрибутивное многообразие конечной сигнатуры, обладающее свойством СЕР, причем подалгебры подпрямо неразложимых алгебр финитно неразложимы. Тогда:

(1) если $V$ - многообразие полурешеточно упорядоченных алгебр, имеющее SEP, то существует алгоритм, позволяющий для любой конечной алгебры $\mathbf{A} \in V$ определить, обладает ли многообразие $V(\mathbf{A})$ свойством SupAP;

(2) если $V$ имеет SEP1, то существует алгоритм, позволяющий для любой конечной алгебры $\mathbf{A} \in V$ определить, обладает ли многообразие $V(\mathbf{A})$ свойством $\mathrm{Str} A \mathrm{P}$.

ДоказАтельство. (1) Сразу следует из теорем 3.7, 5.3, 5.4.

(2) Следует из теорем 3.14, 5.3, 5.5.

Теорема 5.3 применима, например, к многообразиям дистрибутивных решеток с псевдодополнениями, резидуальных решеток [40] и так называемых BL-алгебр [13], используемых для интерпретации нечетких логик, поскольку все эти многообразия конгруэнц-дистрибутивны и обладают свойством СЕР. Все теоремы 5.3-5.6 применимы к многообразиям булевых алгебр с операторами [44].

\section{Список литературы}

[1] А. И. Мальцев, "О некоторых пограничных вопросах алгебры и логики”, Труды Международного конгресса математиков (Москва, 1966), Мир, М., 1966, 217-231.

[2] А. И. Мальцев, Алгебраические системь, Современная алгебра, Наука, М., 1970.

[3] W. Craig, "Three uses of the Herbrand-Gentzen theorem in relating model theory and proof theory", J. Symbolic Logic, 22:3 (1957), 269-285.

[4] E. W. Beth, "On Padoa's method in the theory of definition", Indagationes Math., 15:4 (1953), 330-339.

[5] B. Jónsson, "146-157", Theory of Models (Berkeley, 1963), North-Holland, Amsterdam, 1965.

[6] D. Pigozzi, "Amalgamation, congruence-extension, and interpolation properties in algebras", Algebra Universalis, 1 (1971), 269-349.

[7] J. Barwise, S. Feferman (eds.), Model-Theoretic Logics, Perspect. Math. Logic, Springer-Verlag, New York, NY, 1985.

[8] H. Ono, "Interpolation and the Robinson property for logics not closed under the Boolean operations", Algebra Universalis, 23:2 (1986), 111-122.

[9] I. Sain, "Beth's and Craig's properties via epimorphisms and amalgamation in algebraic logic", Algebraic Logic and Universal Algebra in Computer Science (Ames, IA, 1988), Lecture Notes in Comput. Sci., 425, Springer-Verlag, 1990, 209-225.

[10] J. Czelakowski, D. Pigozzi, "Amalgamation and Interpolation in abstract algebraic logic", Models, Algebras and Proofs (Bogotá, 1995), Lecture Notes in Pure and Appl. Math., 203, Marcel Dekker, New York, NY, 1999, 187-265. 
[11] E. Hoogland, Definability and Interpolation. Model-Theoretic Investigations, ILLC Publications, Dissertation Series, Nr. DS-2001-05, Amsterdam, Univ. of Amsterdam, 2001.

[12] Л. Л. Максимова, “Ограниченная интерполяция и проективное свойство Бета в эквациональной логике", Алгебра и логика, 42:6 (2003), 712-726.

[13] F. Montagna, "Interpolation and Beth's property in propositional many-valued logics: A semantic investigation", Ann. Pure Appl. Logic, 141:1-2 (2006), 148-179.

[14] Л.Л. Максимова, "Слабая форма интерполяции в эквациональной логике”, Алгебра и логика, 47:1 (2008), 94-107.

[15] D. M. Gabbay, L. Maksimova, Interpolation and Definability. Modal and Intuitionistic Logics, Oxford Logic Guides, 46, Clarendon Press, Oxford, 2005.

[16] L. Maksimova, "Definability and interpolation in non-classical logics", Studia Logica, 82:2 (2006), $271-291$.

[17] H. Kihara, H. Ono, "Interpolation properties, Beth definability properties and amalgamation properties for substructural logics", J. Logic Comput., 20:4 (2010), 823-875.

[18] P. D. Bacsich, "Amalgamation properties and interpolation theorems for equational theories", Algebra Universalis, 5:1 (1975), 45-55.

[19] E. W. Kiss, L. Márki, P. Pröhle, W. Tholen, "Categorical algebraic properties. A compendium on amalgamation, congruence extension, epimorphisms, residual smallness, and injectivity", Studia Sci. Math. Hungar., 18:1 (1983), 79-141.

[20] H. Andréka, I. Németi, I. Sain, "Algebraic logic", Handbook of Philosophical Logic, v. 2, Kluwer Acad. Publ., Dordrecht, 2001, 133-247.

[21] Л. Л. Максимова, Е. Орловска, “Свойство Бета и интерполяция в алгебрах и логиках, основанных на решётках", Алгебра и логика, 47:3 (2008), 307-334.

[22] G. Grätzer, General Lattice Theory, Akademie-Verlag, Berlin, 1978.

[23] G. Grätzer, H. Lakser, "The structure of pseudocomplemented distributive lattices. II. Congruence extension and amalgamation", Trans. Amer. Math. Soc., 156 (1971), 343-358.

[24] Л. Л. Максимова, "Теорема Крейга в суперинтуиционистских логиках и амальгамируемые многообразия псевдобулевых алгебр", Алгебра и логика, 16:6 (1977), 643-681.

[25] Л. Л. Максимова, "Модальные логики и многообразия модальных алгебр: свойство Бета, интерполяция и амальгамируемость", Алгебра и логика, 31:2 (1992), 145-166.

[26] Л. Л. Максимова, "Проективные свойства Бета в модальных и суперинтуиционистских логиках", Алгебра и логика, 38:3 (1999), 316-333.

[27] Л. Л. Максимова, "Интерполяционные теоремы в модальных логиках и амальгамируемые многообразия топобулевых алгебр", Алгебра и логика, 18:5 (1979), 556-586.

[28] L. Maksimova, "Interpolation and joint consistency", We Will Show Them!, v. 2, Essays in Honour of Dov Gabbay, King's College Publ., London, 2005, 293-305.

[29] Л. Л. Максимова, “Аналог теоремы Бета в нормальных расширениях модальной логики К4”, Сиб. матем. журн., 33:6 (1992), 118-130.

[30] L. Maksimova, "Problem of restricted interpolation in superintuitionistic and some modal logics", Log. J. IGPL, 18:3 (2010), 367-380.

[31] Л.Л. Максимова, "Проективное свойство Бета и интерполяция в позитивных и близких к ним логиках", Алгебра и логика, 45:1 (2006), 85-113.

[32] L. L. Maksimova, "Intuitionistic logic and implicit definability", Ann. Pure Appl. Logic, 105:1-3 (2000), 83-102.

[33] Л. Л. Максимова, "Неявная определимость и позитивные логики”, Алгебра и логика, 42:1 (2003), $65-93$.

[34] L. Maksimova, "Projective Beth property in Extensions of Grzegorczyk logic", Studia Logica, 83:1-3 (2006), 365-391.

[35] А. В. Карпенко, "Слабое интерполяционное свойство в расширениях логик $S 4$ и $K 4$ ", Алгебра u логика, 47:6 (2008), 705-722.

[36] Л. Л. Максимова, "Разрешимость проективного свойства Бета в многообразиях гейтинговых алгебр", Алгебра и логика, 40:3 (2001), 290-301. 
[37] L. Maksimova, "Decidability of some properties in tabular logics", 13th International Congress of Logic, Methodology and Philosophy of Science, Volume of Abstracts (2007, Beijing, China), 124-125.

[38] A. Wroński, "On a form of equational interpolation property", Foundations of Logic and Linguistics (Salzburg, 1983), Plenum Press, London, 1985, 23-29.

[39] L. Henkin, J. D. Monk, A. Tarski, Cylindric Algebras, Part II, Stud. Logic Found. Math., 115, North-Holland, Amsterdam, 1985.

[40] T. Kowalski, H. Ono, "Splittings in the variety of residuated lattices", Algebra Universalis, 44:3-4 (2000), 283-298.

[41] L. Maksimova, "Restricted interpolation in modal logics", Advances in Modal Logic, v.4, King's College Publ., London, 2003, 297-311.

[42] Л.Л. Максимова, "Интерполяционные теоремы в модальных логиках. Достаточные условия", Алгебра и логика, 19:2 (1980), 194-213.

[43] B. Jónsson, "Algebras whose congruence structures are distributive", Math. Scand., 21 (1967), 110-121.

[44] B. Jónsson, A. Tarski, "Boolean algebras with operators. Part I", Amer. J. Math., 73:4 (1951), 891-939. 